Japan. J. Math.

Vol. 9, No. 2, 1983

\title{
On regularity of positive linear functionals
}

\author{
By Atushi Inoue
}

(Received August 27, 1982)

\section{$\S 1$. Introduction}

In this paper we study positive linear functionals $f$ on *-algebras, by investigating the relation between the operator-representation $\pi_{f}$ and the vector-representation $\lambda_{f}$ generated by the well-known GNS-construction of $f$. The primary purpose is to answer the following question: When is a positive

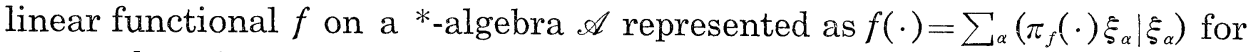
some subset $\left\{\xi_{\alpha}\right\}$ of the domain $\mathscr{D}\left(\pi_{f}\right)$ of $\pi_{f}$ ?

Most study of positive linear functionals $f$ is for the case that $\pi_{f}$ are bounded. However, the operator-representation $\pi_{f}$ is in general unbounded. In the paper [22], Powers investigated unbounded representations of $*_{\text {- }}$ algebras and pointed out that there exists a pathological relation between subrepresentations and commutants, which does not occur in the bounded case. To remove such a phenomenon he introduced the notion of self-adjoint representation. After that, unbounded representations have been studied by many mathematicians $[9,12,13,14,15,16,22,25]$. We here treat positive linear functionals $f$ satisfying a condition weaker than the self-adjointness of $\pi_{f}$.

The nature of the study of positive linear functionals on $*$-algebras $\mathscr{A}$ depends on whether $\mathscr{A}$ has identity or not. When a $*$-algebra $\mathscr{A}$ has identity $e$, our question is solved; in fact, every positive linear functional $f$ on $\mathscr{A}$ is represented as $f(x)=\left(\pi_{f}(x) \lambda_{f}(e) \mid \lambda_{f}(e)\right)$ for all $x \in \mathscr{A}$. We remark that by considering the positive invariant sesquilinear form $f^{\circ}$ defined by $f^{\circ}(x, y)$ $=f\left(y^{*} x\right)$ for a positive linear functional $f$, the study of positive invariant sesquilinear forms is seen to be more general than that of positive linear functionals when *-algebras don't possess identity.

With this fact in mind we study positive invariant sesquilinear forms $\phi$

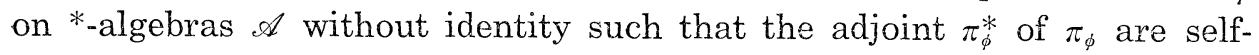
adjoint representations.

We proceed along the following plan.

In Section 2, we introduce some notions which are used in this paper, for example, $O_{p}^{*}$-algebras, positive invariant sesquilinear forms and so on. 
In Section 3, we introduce a $T^{+}$-algebra $\mathfrak{U}_{\phi}$ for a positive invariant sesquilinear form $\phi$, which is an unbounded generalization of the notion of observable algebras established by Tomita [28]. We define a commutant $\mathfrak{H}_{\phi}^{\prime}$ of $\mathfrak{U}_{\phi}$ and show that $\mathfrak{U}_{\phi}^{\prime}$ is a ${ }^{*}$-algebra, and there exist a ${ }^{*}$-representation $\pi$ of $\mathfrak{Y}_{\phi}^{\prime}$ into the Power's commutant $\pi_{\phi}(\mathscr{A})^{\prime}$ of $\pi_{\phi}$ and a linear map $\lambda$ of $\mathfrak{Y}_{\phi}^{\prime}$ into the Hilbert space $\mathfrak{S}_{\phi}$ satisfying that $\lambda(S K)=\pi(S) \lambda(K)$ for each $S, K \in \mathfrak{R}_{\phi}^{\prime}$. By using the *-algebras $\mathfrak{H}_{\phi}$ and $\mathfrak{H}_{\phi}^{\prime}$ we investigate positive invariant sesquilinear forms $\phi$ in the latter section.

In Section 4, we investigate under what conditions a positive invariant sesquilinear form $\phi$ on a ${ }^{*}$-algebra $\mathscr{A}$ is represented as $\phi(x, y)=\left(\pi_{\phi}^{*}(x) \xi \mid \pi_{\phi}^{*}(y) \xi\right)$ for some $\xi \in \mathscr{D}\left(\pi_{\phi}^{*}\right)$. Such a form $\phi$ is called strongly regular. We show that $\phi$ is strongly regular if and only if $\pi\left(\mathfrak{Y}_{\phi}^{\prime}\right)$ is a $C^{*}$-algebra.

In Section 5, we introduce the notion of regularity (resp. $\Sigma$-regularity) of a positive invariant sesquilinear form $\phi$ on a $*$-algebra $\mathscr{A}$ as a natural generalization of the notion of strong regularity; that is, $\phi$ is represented as $\phi(x, y)=\lim _{\alpha}\left(\pi_{\phi}^{*}(x) \xi_{\alpha} \mid \pi_{\phi}^{*}(y) \xi_{\alpha}\right)\left(\operatorname{resp} . \phi(x, y)=\sum_{\alpha}\left(\pi_{\phi}^{*}(x) \xi_{\alpha} \mid \pi_{\phi}^{*}(y) \xi_{\alpha}\right)\right)$ for some net $\left\{\xi_{\alpha}\right\}$ in $\mathscr{D}\left(\pi_{\phi}^{*}\right)$. We show that $\phi$ is regular if and only if $\pi\left(\mathfrak{Y}_{\phi}^{\prime}\right)$ is nondegenerate, and show that if $\pi_{\phi}$ is selfadjoint and $\phi_{E}$ is regular for each non-zero projection $E$ in $\pi_{\phi}(\mathscr{A})^{\prime}$, where $\phi_{E}(x, y)=\left(E \lambda_{\phi}(x) \mid \lambda_{\phi}(y)\right)$ for $x, y \in \mathscr{A}$, then there exists a subset $\left\{\xi_{a}\right\}$ in $\mathscr{D}\left(\pi_{\phi}\right)$ such that $\left(\pi_{\phi}(x) \xi_{\alpha} \mid \pi_{\phi}(y) \xi_{\beta}\right)=0$ for each $x, y \in \mathscr{A}$ and $\alpha \neq \beta$, and $\phi(x, y)=\sum_{\alpha}\left(\pi_{\phi}(x) \xi_{\alpha} \mid \pi_{\phi}(y) \xi_{\alpha}\right)$ for each $x, y \in \mathscr{A}$. We remark that if $\mathscr{A}$ is commutative then $\phi$ is regular if and only if $\phi$ is $\Sigma$-regular.

In Section 6, we give a decomposition theorem for a positive invariant sesquilinear form. We introduce the notion of singularity of positive invariant sesquilinear forms, and show that every positive invariant sesquilinear form $\phi$ is decomposed into the sum $\phi=\phi_{r}+\phi_{s}$ of the regular part $\phi_{r}$ and the singular part $\phi_{s}$. In particular, we show that if $\mathscr{A}$ is commutative then the above decomposition is unique.

In Section 7, we apply our study of positive invariant sesquilinear forms to of weights on $C^{*}$-algebras. For a weight $\phi$ on a $C^{*}$-algebra $\mathscr{A}$ a positive invariant sesquilinear form $\phi^{\circ}$ on $\mathfrak{N}_{\phi} \times \mathfrak{N}_{\phi}$ is defined by $\phi^{\circ}(x, y)=\phi\left(y^{*} x\right)$ for $x, y \in \mathfrak{N}_{\phi}$, where $\mathfrak{N}_{\phi}=\left\{x \in \mathscr{A} ; \phi\left(x^{*} x\right)<\infty\right\}$. By using the results of Combes [4] and Pedersen, Takesaki [21], we show that the regularity and $\Sigma$-regularity of $\phi^{\circ}$ are equivalent.

The author would like to express his sincere gratitude to Professor M. Tomita from whom he learned observable algebras in a series of lectures at Kyushu University in 1978-1979. He also would like to thank Professor Y. Nakagami and Dr. S. Ôta for many comments and suggestions made during the course of preparing the manuscript. 


\section{§ 2. Preliminaries}

In this section we state some definitions and results concerning unbounded operator algebras and positive invariant sesquilinear forms.

Let $\mathscr{D}$ be a dense subspace in a Hilbert space $\mathfrak{F}$. We denote by $\mathscr{L}(\mathscr{D})$ the set of all linear operators from $\mathscr{D}$ into $\mathscr{D}$ and denote by $\mathscr{L}^{+}(\mathscr{D})$ the set of all elements $A$ of $\mathscr{L}(\mathscr{D})$ such that $\mathscr{D}\left(A^{*}\right) \supset \mathscr{D}$ and $A^{+}=A^{*} / \mathscr{D} \in \mathscr{L}(\mathscr{D})$, where $X / \mathscr{D}$ denotes the restriction of a linear operator $X$ to $\mathscr{D}$. Then $\mathscr{L}^{+}(\mathscr{D})$ is a *-algebra under the usual operations and the involution $A \rightarrow A^{+}$. $A$ subalgebra of $\mathscr{L}(\mathscr{D})$ is said to be an $O_{p}$-algebra on $\mathscr{D}$; and a ${ }^{*}$-subalgebra of $\mathscr{L}^{+}(\mathscr{D})$ is said to be an $O_{p}^{*}$-algebra on $\mathscr{D}$. Let $\mathscr{M}$ be an $O_{p}$-algebra on $\mathscr{D}$ and let $\mathscr{M}_{I}$ be the $O_{p}$-algebra on $\mathscr{D}$ obtained from $\mathscr{M}$ by adjoining the identity operator $I$. If $\mathscr{D}$ is complete under the induced topology $t_{\mathscr{M}}$ generated by the seminorms $\left\{\|\cdot\|_{A} ; A \in \mathscr{M}_{I}\right\}$, where $\|\xi\|_{A}=\|A \xi\|$ for $\xi \in \mathscr{D}$, then $\mathscr{M}$ is called closed. It is known that $\mathscr{M}$ is closed if and only if $\tilde{\mathscr{D}}(\mathscr{M}) \equiv \bigcap_{A \in \mathscr{M}} \mathscr{D}(\bar{A})=\mathscr{D}$, where $\bar{A}$ is the smallest closed extension of $A$. For each $A \in \mathscr{M}$ and $\xi \in \mathscr{D}(\mathscr{M})$ putting

$$
\tilde{A} \xi=\bar{A} \xi \quad \text { and } \quad \tilde{\mathscr{M}}=\{\tilde{A} ; A \in \mathscr{M}\},
$$

$\widetilde{\mathscr{M}}$ becomes a closed $O_{p}$-algebra on $\widetilde{\mathscr{D}}(\mathscr{M})$, which is said to be the closure of $\mathscr{M}$. Let $\mathscr{M}$ be an $O_{p}^{*}$-algebra on $\mathscr{D}$. If $\mathscr{D}_{*}(\mathscr{M}) \equiv \bigcap_{A \in \mathscr{M}} \mathscr{D}\left(A^{*}\right)=\mathscr{D}$, then $\mathscr{M}$ is called self-adjoint. We define a commutant of an $O_{p}^{*}$-algebra $\mathscr{M}$ on $\mathscr{D}$ as follows :

$$
\begin{aligned}
\mathscr{M}^{\prime}=\{K \in \mathscr{B}(\mathscr{S}) ;( & (K A \xi \mid \eta)=\left(K \xi \mid A^{+} \eta\right) \\
& \text { for all } A \in \mathscr{M} \text { and } \xi, \eta \in \mathscr{D}\},
\end{aligned}
$$

where $\mathscr{B}(\mathfrak{S})$ denotes the set of all bounded linear operators on $\mathfrak{F}$. If $K \in \mathscr{M}^{\prime}$, then $K \mathscr{D} \subset \mathscr{D}_{*}(\mathscr{M})$ and $\left(A^{+}\right)^{*} K \xi=K A \xi$ for every $A \in \mathscr{M}$ and $\xi \in \mathscr{D}$. The commutant $\mathscr{M}^{\prime}$ of $\mathscr{M}$ is not necessarily an algebra. However, if $\mathscr{M}$ is self-adjoint, then $\mathscr{M}^{\prime}$ is a von Neumann algebra and $\mathscr{M}^{\prime} \mathscr{D}=\mathscr{D}$ [22].

Let $\mathscr{A}$ be a *-algebra. A homomorphism (resp. *-homomorphism) $\pi$ of $\mathscr{A}$ onto an $O_{p}$-algebra (resp. an $O_{p}^{*}$-algebra) on $\mathscr{D}$ is said to be a representation (resp. a ${ }^{*}$-representation) of $\mathscr{A}$ on $\mathfrak{S}$ with domain $\mathscr{D}(\pi)=\mathscr{D}$. A representation $\pi$ of $\mathscr{A}$ is called closed if the $O_{p}$-algebra $\pi(\mathscr{A})$ is closed; and a *-representation $\pi$ of $\mathscr{A}$ is called self-adjoint if the $O_{p}^{*}$-algebra $\pi(\mathscr{A})$ is self-adjoint. Let $\pi, \rho$ be representations of $\mathscr{A}$ on a Hilbert space $\mathfrak{F}$. If $\mathscr{D}(\rho) \subset \mathscr{D}(\pi)$ and $\rho(x) \xi$ $=\pi(x) \xi$ for all $x \in \mathscr{A}$ and $\xi \in \mathscr{D}(\rho)$, then we say that $\pi$ is an extension of $\rho$, which is denoted by $\rho \subset \pi$. Let $\pi$ be a representation of $\mathscr{A}$ on a Hilbert space $\mathfrak{c}$. Then we define closed representations of $\mathscr{A}$ as follows:

$$
\mathscr{D}(\tilde{\pi})=\bigcap_{x \in \mathscr{A}} \mathscr{D}(\overline{\pi(x)}), \quad \tilde{\pi}(x)=\overline{\pi(x)} / \mathscr{D}(\tilde{\pi}) ;
$$




$$
\mathscr{D}\left(\pi^{*}\right)=\bigcap_{x \in \mathscr{A}} \mathscr{D}\left(\pi(x)^{*}\right), \quad \pi^{*}(x)=\pi\left(x^{*}\right)^{*} / \mathscr{D}\left(\pi^{*}\right)
$$

for $x \in \mathscr{A}$. If $\mathscr{D}\left(\pi^{*}\right)$ is dense in $\mathscr{S}_{\mathrm{C}}$, then the closed representation $\pi^{* *}$ of $\mathscr{A}$ is defined by

$$
\mathscr{D}\left(\pi^{* *}\right)=\bigcap_{x \in \mathscr{A}} \mathscr{D}\left(\pi^{*}(x)^{*}\right), \quad \pi^{* *}(x)=\pi^{*}\left(x^{*}\right)^{*} / \mathscr{D}\left(\pi^{* *}\right)
$$

for $x \in \mathscr{A}$. Then we see that if $\pi$ is a $*$-representation, then $\tilde{\pi}$ and $\pi^{* *}$ are *-representations satisfying that $\pi \subset \pi \subset \pi^{* *} \subset \pi^{*}$ and $\pi^{* *}(\mathscr{A})^{\prime}=\pi(\mathscr{A})^{\prime}$. However, $\pi^{*}$ is not necessarily a $*$-representation. We remark that there exist *-representations $\pi_{1}, \pi_{2}$ such that $\tilde{\pi}_{1} \subseteq \pi_{1}^{* *}=\pi_{1}^{*}$ and $\tilde{\pi}_{2}=\pi_{2}^{* *} \subseteq \pi_{2}^{*}[9,15]$.

Let $\mathscr{A}$ be a $*$-algebra. A sesquilinear form $\phi$ of $\mathscr{A} \times \mathscr{A}$ into the complex number field $C$ is called positive invariant if

$$
\phi(x, x) \geqq 0 \quad \text { and } \quad \phi(x y, z)=\phi\left(y, x^{*} z\right)
$$

for each $x, y, z \in \mathscr{A}$. Let $\phi$ be a positive invariant sesquilinear form on $\mathscr{A} \times \mathscr{A}$. It is easily seen that $N_{\phi}=\{x \in \mathscr{A} ; \phi(x, x)=0\}$ is a left ideal in $\mathscr{A}$. For each $x \in \mathscr{A}$ we denote by $\lambda_{\phi}(x)$ the coset of $\mathscr{A} / N_{\phi}$ which contains $x$, and define by

$$
\left(\lambda_{\phi}(x) \mid \lambda_{\phi}(y)\right)=\phi(x, y)
$$

an inner product on $\lambda_{\phi}(\mathscr{A})$. Let $\mathfrak{S}_{\phi}$ be the Hilbert space which is the completion of the pre-Hilbert space $\lambda_{\phi}(\mathscr{A})$. We define a ${ }^{*}$-representation $\pi_{\phi}^{\circ}$ of $\mathscr{A}$ on $\tilde{S}_{\phi}$ with domain $\lambda_{\phi}(\mathscr{A})$ by

$$
\pi_{\phi}^{\circ}(x) \lambda_{\phi}(y)=\lambda_{\phi}(x y)
$$

for $x, y \in \mathscr{A}$. We denote by $\pi_{\phi}$ the closure $\tilde{\pi}_{\phi}^{\circ}$ of $\pi_{\phi}^{\circ}$; that is, $\pi_{\phi}$ is a closed *-representation of $\mathscr{A}$ on $\mathscr{\mathscr { C }}_{\phi}$ with domain $\mathscr{D}\left(\pi_{\phi}\right)=\bigcap_{x \in \mathscr{A}} \mathscr{D}\left(\overline{\left.\pi_{\phi}^{\circ}(x)\right)}\right.$. We call $\left(\pi_{\dot{\phi}}, \lambda_{\dot{\phi}}, \mathscr{D}\left(\pi_{\dot{\phi}}\right), \mathscr{S}_{\dot{\phi} \phi}\right)$ the GNS-construction of $\phi$. If $\pi_{\dot{\phi}}$ is bounded; that is, $\pi_{\dot{\phi}}(\mathscr{A})$ $\subset \mathscr{B}\left(\mathfrak{S}_{\phi}\right)$, then $\phi$ is called admissible; and if $\pi_{\phi}$ is selfadjoint, then $\phi$ is said to be a Riesz form. We note that every admissible positive invariant sesquilinear form is a Riesz form. Examples of Riesz forms have appeared in [2, 3, $6,12,15,19,22]$.

Let $f$ be a linear functional on a $*$-algebra $\mathscr{A}$. If $f\left(x^{*} x\right) \geqq 0$ for each $x \in \mathscr{A}$, then $f$ is called positive. Let $f$ be a positive linear functional on $\mathscr{A}$. We define a positive invariant sesquilinear form $f^{\circ}$ on $\mathscr{A} \times \mathscr{A}$ by

$$
f^{\circ}(x, y)=f\left(y^{*} x\right)
$$

for $x, y \in \mathscr{A}$, which is said to be the positive invariant sesquilinear form generated by $f$. We simply denote by $\left(\pi_{f}, \lambda_{f}, \mathscr{D}\left(\pi_{f}\right), \mathscr{S}_{f}\right)$ the GNS-construction of $f^{\circ}$. A positive linear functional $f$ on $\mathscr{A}$ is called admissible (resp. a Riesz 
functional) if $f^{\circ}$ is admissible (resp. a Riesz form).

\section{§3. Unbounded observable algebras}

Let $\mathscr{D}$ be a dense subspace of a Hilbert space $\mathfrak{F _ { \mathcal { C } }}$. For each $\xi \in \mathfrak{F}$ we define a continuous linear functional $\xi^{*}$ on $\mathfrak{S}$ by $\eta \in \mathfrak{S} \rightarrow(\eta \mid \xi) \in C$. For a subspace $\mathfrak{M}$ of $\mathfrak{S}$ the map $\xi \rightarrow \xi^{*}$ is a conjugate linear isometry of $\mathfrak{M}$ onto $\mathfrak{M}^{*}$ $=\left\{\xi^{*} ; \xi \in \mathfrak{M}\right\}$. We define on the product space $T^{+}(\mathscr{D})=\mathscr{L}^{+}(\mathscr{D}) \times \mathscr{D} \times \mathscr{D}^{*}$ a multiplication and an involution as follows:

$$
\begin{aligned}
\left(S_{0}, \xi_{1}, \xi_{2}^{*}\right)\left(T_{0}, \eta_{1}, \eta_{2}^{*}\right) & =\left(S_{0} T_{0}, S_{0} \eta_{1},\left(T_{0}^{+} \xi_{2}\right)^{*}\right), \\
\left(S_{0}, \xi_{1}, \xi_{2}^{*}\right)^{+} & =\left(S_{0}^{+}, \xi_{2}, \xi_{1}^{*}\right) .
\end{aligned}
$$

Then $T^{+}(\mathscr{D})$ is a *algebra. For each $S=\left(S_{0}, \xi_{1}, \xi_{2}^{*}\right) \in T^{+}(\mathscr{D})$ we put

$$
\pi(S)=S_{0}, \quad \lambda(S)=\xi_{1}, \quad \lambda^{*}(S)=\xi_{2}^{*} .
$$

DeFINITION 3.1. A *-subalgebra of $T^{+}(\mathscr{D})$ is said to be an unbounded observable algebra on $\mathscr{D}$ (simply, a $T^{+}$-algebra on $\mathscr{D}$ ).

Let $\mathfrak{A}$ be a $T^{+}$-algebra on $\mathscr{D}$. We note that $\pi$ is a ${ }^{*}$-representation of $\mathscr{U}$ on $\mathscr{S}$ with domain $\mathscr{D}, \lambda$ is a linear map of $\mathscr{X}$ into $\mathscr{D}$ satisfying $\lambda(A B)=\pi(A) \lambda(B)$ and $\lambda\left(A^{+}\right)=\lambda^{*}(A)^{*}$, and $\lambda^{*}$ is a conjugate linear map of $\mathfrak{A}$ into $\mathscr{D}^{*}$ satisfying $\lambda^{*}(A B)\left(\pi(B)^{+} \lambda\left(A^{+}\right)\right)^{*}$ for each $A, B \in \mathfrak{U}$.

Definition 3.2. Let $\mathfrak{U}$ be a $T^{+}$-algebra on $\mathscr{D}$. If the *-representation $\pi$ is closed (resp. self-adjoint), then $\mathfrak{V}$ is called $\pi$-closed (resp. $\pi$-self-adjoint).

REMARK. When $\mathscr{D}=\mathfrak{S}_{\mathcal{C}}, T^{+}(\mathfrak{S})$ is a Banach *algebra equipped with the norm:

$$
\|T\|=\max \left(\|\pi(T)\|,\|\lambda(T)\|,\left\|\lambda\left(T^{+}\right)\right\|\right) .
$$

Tomita has called a *-sublgebra of $T^{+}(\mathfrak{S})$ an observable algebra on $\mathfrak{S}$ (simply,

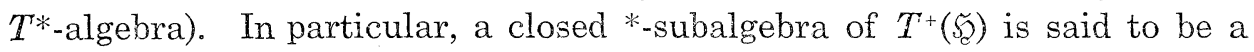
$C T^{*}$-algebra.

Definition 3.3. Let $\mathfrak{U}$ be a $T^{+}$-algebra on $\mathscr{D}$. We define a commutant of $\mathfrak{U}$ as follows:

$$
\begin{aligned}
\mathfrak{U}^{\prime}= & \left\{K=\left(K_{0}, \xi, \eta^{*}\right) \in \pi(\mathfrak{V})^{\prime} \times \mathscr{D}\left(\pi^{*}\right) \times \mathscr{D}\left(\pi^{*}\right)^{*} ;\right. \\
& \left.K_{0} \lambda(A)=\pi\left(A^{+}\right)^{*} \xi, K_{0}^{*} \lambda(A)=\pi\left(A^{+}\right)^{*} \eta \text { for all } A \in \mathfrak{U}\right\} .
\end{aligned}
$$

For each $K=\left(K_{0}, \xi, \eta^{*}\right) \in \mathfrak{Y}^{\prime}$ we put

$$
\pi(K)=K_{0}, \quad \lambda(K)=\xi, \quad \lambda^{*}(K)=\eta^{*} .
$$


The commutant $\mathfrak{U}^{\prime}$ is a vector space, which is invariant under the involution $K=\left(\pi(K), \lambda(K), \lambda^{*}(K)\right) \rightarrow K^{*}=\left(\pi(K)^{*}, \lambda^{*}(K)^{*}, \lambda(K)^{*}\right)$. But, $\mathfrak{Y}^{\prime}$ is not necessarily a $T^{*}$-algebra.

Proposition 3.4. If $\mathfrak{U}$ is a $T^{+}$-algebra on $\mathscr{D}$ satisfying $\pi^{*}=\pi^{* *}$ (in particular, $\mathfrak{Y}$ is $\pi$-selfadjoint), then $\mathfrak{Y}^{\prime}$ is a $C T^{*}$-algebra and $\pi\left(\mathfrak{U}^{\prime}\right)$ is a ${ }^{*}$-subalgebra of the von Neumann algebra $\pi(\mathfrak{H})^{\prime}$.

Proof. Since $\pi^{*}$ is a self-adjoint representation of $\mathscr{U}$ with domain $\mathscr{D}\left(\pi^{*}\right)$ and $\pi^{*}(\mathfrak{U})^{\prime}=\pi^{* *}(\mathfrak{U})^{\prime}=\pi(\mathfrak{U})^{\prime}$, it follows that $\pi(\mathfrak{U})^{\prime}$ is a von Neumann algebra, $\pi(\mathfrak{U})^{\prime} \mathscr{D}\left(\pi^{*}\right)=\mathscr{D}\left(\pi^{*}\right)$ and $C \pi^{*}(A) \xi=\pi^{*}(A) C \xi$ for each $A \in \mathfrak{A}, C \in \pi(\mathfrak{H})^{\prime}$ and $\xi \in$ $\mathscr{D}\left(\pi^{*}\right)$, which implies that $\mathfrak{U}^{\prime}$ is a $C T^{*}$-algebra.

Let $\mathscr{A}$ be a ${ }^{*}$-algebra and $\phi$ be a positive invariant sesquilinear form on $\mathscr{A} \times \mathscr{A}$. We put

$$
\mathfrak{A}_{\phi}=\left\{\left(\pi_{\phi}(x), \lambda_{\phi}(x), \lambda_{\phi}\left(x^{*}\right)^{*}\right) ; x \in \mathscr{A}\right\} .
$$

Then $\mathfrak{I}_{\phi}$ is a $\pi$-closed $T^{+}$-algebra on $\mathscr{D}\left(\pi_{\phi}\right)$, which is said to be the $T^{+}$-algebra generated by $\phi$. Let $f$ be a positive linear functional on $\mathscr{A}$. We denote by $\mathfrak{U}_{f}$ the $T^{+}$-algebra generated by $f^{\circ}$.

In this paper we shall study positive invariant sesquilinear forms $\phi$ by using the $T^{+}$-algebra $\mathfrak{A}_{\phi}$ and the commutant $\mathfrak{Y}_{\phi}^{\prime}$.

\section{$\S 4$. Strongly regular positive invariant sesquilinear forms}

Throughout this section let $\mathscr{A}$ be a $*$-algebra without identity. We investigate under what conditions a positive invariant sesquilinear form $\phi$ on $\mathscr{A} \times \mathscr{A}$ is represented as $\phi(x, y)=\left(\pi_{\phi}^{*}(x) \xi \mid \pi_{\phi}^{*}(y) \xi\right)$ for some $\xi \in \mathscr{D}\left(\pi_{\phi}^{*}\right)$.

DEFINITION 4.1. Let $\phi$ be a positive invariant sesquilinear form on $\mathscr{A} \times \mathscr{A}$. A positive invariant sesquilinear form $\psi$ on $\mathscr{A} \times \mathscr{A}$ is called $\phi$ dominated if $\psi \leqq \gamma \phi$ for some positive unmber $\gamma$; that is, $\psi(x, x) \leqq \gamma \phi(x, x)$ for all $x \in \mathscr{A}$. A positive linear functional $f$ on $\mathscr{A}$ is called $\phi$-majorized if $f^{\circ}$ is $\phi$-dominated and there is a constant $\gamma$ such that $|f(x)|^{2} \leqq \gamma \phi(x, x)$ for all $x \in \mathscr{A}$.

LEMMA 4.2. Let $\phi$ and $\psi$ be positive invariant sesquilinear forms on $\mathscr{A} \times \mathscr{A}$ and $f$ be a positive linear functional on $\mathscr{A}$. Then the following statements hold.

(1) $\psi$ is $\phi$-dominated if and only if there exists a positive operator $K_{0}$ in $\pi_{\phi}(\mathscr{A})^{\prime}$ such that $\psi(x, y)=\left(K_{0} \lambda_{\phi}(x) \mid \lambda_{\phi}(y)\right)$ for all $x, y \in \mathscr{A}$.

(2) $f$ is $\phi$-majorized if and only if there exists an element $K$ of $\mathfrak{Y}_{\phi}^{\prime}$ such that $\pi(K) \geqq 0$ and $f(x)=\left(\lambda_{\phi}(x) \mid \lambda(K)\right)$ for all $x \in \mathscr{A}$.

Proof. (1) Suppose that $\psi$ is $\phi$-dominated. Then the map $\lambda_{\phi}(x) \rightarrow \lambda_{\psi}(x)$ 
is extended to a continuous linear map $S_{0}$ of $\mathscr{F}_{\dot{\phi}}$ into $\mathscr{F}_{\psi}$. We put $K_{0}=S_{0}^{*} S_{0}$. Then it is clear that $K_{0} \in \pi_{\dot{\phi}}(\mathscr{A})^{\prime}$ and $\psi(x, y)=\left(K_{0} \lambda_{\dot{\phi}}(x) \mid \lambda_{\dot{\phi}}(y)\right)$ for all $x, y \in \mathscr{A}$. The converse is trivial.

(2) Suppose that $f$ is $\phi$-majorized. Then the map $\lambda_{\phi}(x) \rightarrow f(x)$ is extended to a continuous linear functional on $\mathscr{S}_{\dot{\phi}}$. By the Riesz theorem there exists an element $\xi$ of $\mathfrak{S}_{\dot{\phi}}$ such that $f(x)=\left(\lambda_{\phi}(x) \mid \xi\right)$ for all $x \in \mathscr{A}$. We now have

$$
\left|\left(\pi_{\dot{\phi}}\left(x^{*}\right) \lambda_{\phi}(y) \mid \xi\right)\right|^{2}=\left|f\left(x^{*} y\right)\right|^{2} \leqq f\left(x^{*} x\right) f\left(y^{*} y\right) \leqq \gamma f\left(x^{*} x\right)\left\|\lambda_{\phi}(y)\right\|^{2}
$$

for all $x, y \in \mathscr{A}$, and hence $\xi \in \mathscr{D}\left(\pi_{\phi}^{*}\right)$ and $f\left(x^{*} y\right)=\left(\lambda_{\dot{\phi}}(y) \mid \pi_{\phi}^{*}(x) \xi\right)$ for all $x, y \in \mathscr{A}$. Since $f^{\circ}$ is $\phi$-dominated, it follows from (1) that there exists a positive operator $K_{0}$ in $\pi_{\phi}(\mathscr{A})^{\prime}$ such that $f\left(x^{*} y\right)=\left(\lambda_{\phi}(y) \mid K_{0} \lambda_{\phi}(x)\right)$ for all $x, y \in \mathscr{A}$. Hence, we have $K_{0} \lambda_{\phi}(x)=\pi_{\phi}^{*}(x) \xi$ for all $x \in \mathscr{A}$, which implies that $K=\left(K_{0}, \xi, \xi^{*}\right) \in \mathfrak{X}_{\phi}^{\prime}$ and $f(x)=\left(\lambda_{\phi}(x) \mid \lambda(K)\right)$ for all $x \in \mathscr{A}$.

Definttion 4.3. A positive invariant sesquilinear form $\phi$ on $\mathscr{A} \times \mathscr{A}$ is called strongly regular if there exists a $\phi$-majorized positive linear functional $f$ on $\mathscr{A}$ such that $f^{\circ}=\phi$. A positive linear functional $f$ on $\mathscr{A}$ is called strongly regular if $f^{\circ}$ is strongly regular.

THEOREM 4.4. Let $\phi$ be a positive invariant sesquilinear form on $\mathscr{A} \times \mathscr{A}$. Consider the following statements.

(1) $\phi$ is strongly regular.

(2) There exists an element $\xi$ of $\mathscr{D}\left(\pi_{\phi}^{*}\right)$ such that $\lambda_{\dot{\phi}}(x)=\pi_{\phi}^{*}(x) \xi$ for all $x \in \mathscr{A}$.

(3) $I \in \pi\left(\mathfrak{A}_{\dot{\phi}}^{\prime}\right)$.

(4) There exists an element $\xi$ of $\mathscr{D}\left(\pi_{\phi}^{*}\right)$ such that $\phi(x, y)=\left(\pi_{\phi}^{*}(x) \xi \mid \pi_{\phi}^{*}(y) \xi\right)$ for all $x, y \in \mathscr{A}$.

Then the following implications hold:

$$
(1) \Longleftrightarrow(2) \Longleftrightarrow(3)
$$

If $\pi_{\phi}^{*}$ is a self-adjoint representation (in particular, $\phi$ is a Riesz form), then the above statements (1) (4) are equivalent to the following statements (5), (6) and (7).

(5) $\pi\left(\mathfrak{Y}_{\phi}^{\prime}\right)$ is a nondegenerate *algebra and $\pi(K) \in \pi\left(\mathfrak{Y}_{\phi}^{\prime}\right) \rightarrow \lambda(K) \in{\mathfrak{\mathfrak { C } _ { \phi }}}$ is uniformly continuous.

(6) $\pi\left(\mathfrak{H}_{\phi}^{\prime}\right)$ is a $C^{*}$-algebra.

(7) $\pi_{\phi}(\mathscr{A})^{\prime}=\pi\left(\mathfrak{Y}_{\phi}^{\prime}\right)$.

Proof. $\quad(1) \Rightarrow(3) \quad$ Let $f$ be a $\phi$-majorized positive linear functional on $\mathscr{A}$ with $f^{\circ}=\phi$. By Lemma 4.2 there exists an element $K$ of $\mathfrak{U}_{\phi}^{\prime}$ such that 
$\pi(K) \geqq 0$ and $f(x)=\left(\lambda_{\phi}(x) \mid \lambda(K)\right)$ for all $x \in \mathscr{A}$. Then we have

$$
\begin{aligned}
\left(\lambda_{\phi}(x) \mid \lambda_{\phi}(y)\right)=f\left(y^{*} x\right) & =\left(\lambda_{\phi}(x) \mid \pi_{\phi}^{*}(y) \lambda(K)\right) \\
& =\left(\lambda_{\phi}(x) \mid \pi(K) \lambda_{\phi}(y)\right)
\end{aligned}
$$

for all $x, y \in \mathscr{A}$, and hence $\pi(K)=I$.

Implications $(3) \Rightarrow(2) \Rightarrow(1)$ and $(3) \Rightarrow(4)$ are almost trivial.

Suppose that $\pi_{\phi}^{*}$ is self-adjoint; that is, $\pi_{\phi}^{*}=\pi_{\phi}^{* *}$.

$(4) \Rightarrow(1)$ Suppose that there exists an element $\xi$ of $\mathscr{D}\left(\pi_{\phi}^{*}\right)$ such that $\phi(x, y)=\left(\pi_{\phi}^{*}(x) \xi \mid \pi_{\phi}^{*}(y) \xi\right)$ for all $x, y \in \mathscr{A}$. We put

$$
f(x)=\left(\pi_{\phi}^{*}(x) \xi \mid \xi\right) \quad \text { for } x \in \mathscr{A} .
$$

Since $\xi \in \mathscr{D}\left(\pi_{\phi}^{*}\right)=\mathscr{D}\left(\pi_{\phi}^{* *}\right)$, it follows that $f$ is a $\phi$-majorized positive linear functional on $\mathscr{A}$ with $f^{\circ}=\phi$.

$(2) \Rightarrow(7) \quad$ Suppose that there exists an element $\xi$ of $\mathscr{D}\left(\pi_{\phi}^{*}\right)$ such that $\lambda_{\phi}(x)$ $=\pi_{\phi}^{*}(x) \xi$ for all $x \in \mathscr{A}$. By Proposition $3.4 \pi\left(\mathfrak{U}_{\phi}^{\prime}\right)$ is a ${ }^{*}$-algebra on $\mathscr{F}_{\bar{\phi}}$. Since $\pi_{\phi}^{*}$ is a self-adjoint representation of $\mathscr{A}$ and $\pi_{\phi}^{*}(\mathscr{A})^{\prime}=\pi_{\phi}(\mathscr{A})^{\prime}$, it follows that $\pi_{\dot{\phi}}(\mathscr{A})^{\prime} \mathscr{D}\left(\pi_{\phi}^{*}\right)=\mathscr{D}\left(\pi_{\phi}^{*}\right)$ and $C \pi_{\phi}^{*}(x) \xi=\pi_{\phi}^{*}(x) C \xi$ for each $x \in \mathscr{A}, C \in \pi_{\phi}(\mathscr{A})^{\prime}$ and $\xi \in$ $\mathscr{D}\left(\pi_{\phi}^{*}\right)$. Hence, we have $\left(C, C \xi,\left(C^{*} \xi\right)^{*}\right) \in \mathfrak{H}_{\dot{\phi}}^{\prime}$ for each $C \in \pi_{\dot{\phi}}(\mathscr{A})^{\prime}$, which implies that $\pi_{\phi}(\mathscr{A})^{\prime}=\pi\left(\mathscr{U}_{\phi}^{\prime}\right)$.

$(7) \Rightarrow(6)$ This follows since $\pi_{\dot{\phi}}(\mathscr{A})^{\prime}$ is a von Neumann algebra.

$(6) \Rightarrow(5)$ Since $\pi\left(\mathfrak{Y}_{\phi}^{\prime}\right)$ is a $C^{*}$-algebra, it is nondegenerate. Suppose $\pi(K)$ $=0\left(K \in \mathfrak{P}_{\phi}^{\prime}\right)$. Then, for each $S \in \mathfrak{Y}_{\phi}^{\prime}$ and $x \in \mathscr{A}$ we have

$$
\begin{aligned}
\left(\lambda(K) \mid \pi(S) \lambda_{\phi}(x)\right) & =\left(\lambda(K) \mid \pi_{\phi}^{*}(x) \lambda(S)\right) \\
& =\left(\pi_{\phi}^{*}\left(x^{*}\right) \lambda(K) \mid \lambda(S)\right)\left(\text { by } \pi_{\phi}^{*}=\pi_{\phi}^{* * *}\right) \\
& =\left(\pi(K) \lambda_{\phi}\left(x^{*}\right) \mid \lambda(S)\right) \\
& =0,
\end{aligned}
$$

and hence $\lambda(K)=0$. Hence, $\pi(K) \rightarrow \lambda(K)$ is a linear map of $\pi\left(\mathfrak{U}_{\phi}^{\prime}\right)$ into ${\mathfrak{\mathfrak { C } _ { \phi }}}_{\phi}$. Let $\left\{K_{n}\right\}$ be a sequence in $\mathfrak{r}_{\phi}^{\prime}$ such that $\left\{\pi\left(K_{n}\right)\right\}$ converges uniformly to $K_{0}$ and $\left\{\lambda\left(K_{n}\right)\right\}$ converges to $\xi$. Since $\pi\left(\mathfrak{U}_{\phi}^{\prime}\right)$ is a $C^{*}$-algebra, there exists an element $K$ of $\mathfrak{Y}_{\dot{\phi}}^{\prime}$ such that $K_{0}=\pi(K)$. Furthermore, since

$$
\begin{aligned}
\left(\pi_{\phi}\left(x^{*}\right) \lambda_{\phi}(y) \mid \xi\right) & =\lim _{n \rightarrow \infty}\left(\pi_{\phi}\left(x^{*}\right) \lambda_{\dot{\phi}}(y) \mid \lambda\left(K_{n}\right)\right) \\
& =\lim _{n \rightarrow \infty}\left(\lambda_{\phi}(y) \mid \pi\left(K_{n}\right) \lambda_{\phi}(x)\right) \\
& =\left(\lambda_{\phi}(y) \mid \pi(K) \lambda_{\phi}(x)\right) \\
& =\left(\lambda_{\phi}(y) \mid \pi_{\phi}^{*}(x) \lambda(K)\right)
\end{aligned}
$$

for each $x, y \in \mathscr{A}$, we have $\xi \in \mathscr{D}\left(\pi_{\phi}^{*}\right)$ and $\pi_{\phi}^{*}(x) \xi=\pi_{\phi}^{*}(x) \lambda(K)$ for all $x \in \mathscr{A}$. Since $\pi\left(\mathfrak{H}_{\dot{\phi}}^{\prime}\right)$ is nondegenerate, we have $\xi=\lambda(K)$, so that the linear map $\pi(K)$ 
$\rightarrow \lambda(K)$ is closed. By the closed graph theorem the map $\pi(K) \rightarrow \lambda(K)$ is uniformly continuous.

$(5) \Rightarrow(2)$ Since $\pi(K) \rightarrow \lambda(K)$ is uniformly continuous, there exists a constant $\gamma$ such that $\|\lambda(K)\| \leqq \gamma\|\pi(K)\|$ for all $K \in \mathfrak{Y}_{\phi}^{\prime}$. For each $\varepsilon>0$ and $K_{1}, \cdots$, $K_{n} \in \mathfrak{N}_{\phi}^{\prime}$ we put

$$
\Delta\left(\varepsilon ; K_{1}, \cdots, K_{n}\right)=\left\{\xi \in \mathfrak{S}_{\overline{\mathcal{C}}} ;\|\xi\| \leqq \gamma,\left\|\pi\left(K_{1}\right) \xi-\lambda\left(K_{i}\right)\right\| \leqq \varepsilon \text { for } i=1, \cdots, n\right\} .
$$

Then $\Delta\left(\varepsilon ; K_{1}, \cdots, K_{n}\right)$ is a weakly compact subset of $\tilde{S}_{\phi}$. Since $\pi\left(\mathfrak{U}_{\phi}^{\prime}\right)$ is nondegenerate, we have $\Delta\left(\varepsilon ; K_{i}, \cdots, K_{n}\right) \neq \phi$. Hence, $\bigcap_{\substack{\varepsilon>0 \\ K_{1} \in \mathfrak{H}_{\phi}^{\prime}}} \Delta(\varepsilon ; K) \neq \phi$, which implies that there exists an element $\xi$ of $\mathfrak{F}_{\phi}$ such that $\lambda(K)=\pi(K) \xi$ for all $K \in \mathfrak{U}_{\dot{\phi}}^{\prime}$. Since $\pi\left(\mathfrak{U}_{\phi}^{\prime}\right)$ is nondegenerate, there exists a net $\left\{K_{\alpha}\right\}$ in $\mathfrak{U}_{\phi}^{\prime}$ such that $\left\{\pi\left(K_{\alpha}\right)\right\}$ converges strongly to $I$. Then we have

$$
\begin{aligned}
\lim _{\alpha} \lambda\left(K_{\alpha}\right) & =\lim _{\alpha} \pi\left(K_{\alpha}\right) \xi=\xi, \\
\left(\pi_{\phi}\left(x^{*}\right) \lambda_{\phi}(y) \mid \xi\right) & =\lim _{\alpha}\left(\pi_{\phi}\left(x^{*}\right) \lambda_{\phi}(y) \mid \lambda\left(K_{\alpha}\right)\right) \\
& =\lim _{\alpha}\left(\lambda_{\phi}(y) \mid \pi\left(K_{\alpha}\right) \lambda_{\phi}(x)\right) \\
& =\left(\lambda_{\phi}(y) \mid \lambda_{\phi}(x)\right)
\end{aligned}
$$

for all $x, y \in \mathscr{A}$, and hence $\xi \in \mathscr{D}\left(\pi_{\phi}^{*}\right)$ and $\lambda_{\phi}(x)=\pi_{\phi}^{*}(x) \xi$ for all $x \in \mathscr{A}$. This completes the proof.

Corollary 4.5. Let $f$ be a positive linear functional on $\mathscr{A}$. Consider the following statements.

(1) $f$ is $f^{\circ}$-majorized; that is, there exists a constant $\gamma$ such that $|f(x)|^{2} \leqq$ $\gamma f\left(x^{*} x\right)$ for all $x \in \mathscr{A}$.

(2) There exists an element $\left(I, \xi, \xi^{*}\right)$ of $\mathfrak{Y}_{f}^{\prime}$ such that $f(x)=\left(\pi_{f}^{*}(x) \xi \mid \xi\right)$ for all $x \in \mathscr{A}$.

(3) $f$ is strongly regular.

(4) There exists an element $\xi$ of $\mathscr{D}\left(\pi_{f}^{*}\right)$ such that $\lambda_{f}(x)=\pi_{f}^{*}(x) \xi$ for all $x \in \mathscr{A}$.

Then the following implications hold:

$$
\begin{gathered}
(1) \Longleftrightarrow(2) \\
\Downarrow \\
(3) \Longleftrightarrow(4) .
\end{gathered}
$$

Proof. This is almost similar to the proof of Theorem 4.4.

ExAmples. (1) Every positive invariant sesquilinear form on a *algebra with identity is strongly regular. 
(2) A linear functional $f$ on $\mathscr{A}$ is called hermitian if $f\left(x^{*}\right)=\bar{f}(x)$ for each $x \in \mathscr{A}$. A positive hermitian linear functional $f$ on $\mathscr{A}$ satisfies the condition (1) of Corollary 4.5 if and only if $f$ is extendable; that is, $f$ can be extended to a positive linear functional on $\mathscr{A}_{e}[17,18,23]$. Hence, every extendable positive linear functional is strongly regular.

(3) Let $\phi$ be an admissible positive invariant sesquilinear form on a $*$-algebra $\mathscr{A}$. Then we see that $\phi$ is strongly regular if and only if $\pi_{\phi}(x)$ $\rightarrow \lambda_{\phi}(x)$ is uniformly continuous. This is similar to the proof $(5) \Rightarrow(2)$ of Theorem 4.4.

(4) Let $\mathscr{A}$ be a locally convex *-algebra [1]. A set $\left\{e_{\alpha}\right\}$ in $\mathscr{A}$ is said to be an approximate identity of $\mathscr{A}$ if $\lim _{\alpha} e_{\alpha} x=\lim _{\alpha} x e_{\alpha}=x$ for each $x \in \mathscr{A}$. An approximate identity $\left\{e_{\alpha}\right\}$ of $\mathscr{A}$ is called bounded if $\left\{e_{\alpha}\right\}$ and $\left\{e_{\alpha}^{*} e_{\alpha}\right\}$ are bounded subsets of $\mathscr{A}$. We see that every $B^{*}$-algebra, or more generally, locally convex $G B *$-algebra has a bounded approximate identity [3]. Every continuous positive linear functional $f$ on a locally convex *-algebra with a bounded approximate identity satisfies the condition (1) of Corollary 4.5, and hence $f$ is strongly regular.

\section{§5. $\quad \Sigma$-regular positive invariant sesquilinear forms}

In Section 4 we have investigated under what conditions a positive invariant sesquilinear form $\phi$ is strongly regular; that is, $\phi(x, y)=\left(\pi_{\phi}^{*}(x) \xi \mid\right.$ $\left.\pi_{\phi}^{*}(y) \xi\right)$ for some $\xi \in \mathscr{D}\left(\pi_{\phi}^{*}\right)$. As a natural generalization of the strongly regularity, in this section we investigate under what conditions a positive invariant sesquilinear form $\phi$ is represented as $\phi(x, y)=\sum_{\alpha}\left(\pi_{\phi}^{*}(x) \xi_{\alpha} \mid \pi_{\phi}^{*}(y) \xi_{\alpha}\right)$ for some set $\left\{\xi_{\alpha}\right\}$ in $\mathscr{D}\left(\pi_{\phi}^{*}\right)$.

Throughout this section let $\mathscr{A}$ be a $*$-algebra without identity.

Definition 5.1. A positive invariant sesquilinear form $\phi$ on $\mathscr{A} \times \mathscr{A}$ is called $\Sigma$-regular (resp. regular) if there exists a set $\left\{f_{\alpha}\right\}$ of $\phi$-majorized positive linear functionals on $\mathscr{A}$ such that $\phi(x, y)=\sum_{\alpha} f_{\alpha}^{\circ}(x, y)$ (resp. $f_{\alpha}^{\circ} \leqq \phi$ for each $\alpha$ and $\left.\phi(x, y)=\lim _{\alpha} f_{\alpha}^{\circ}(x, y)\right)$ for all $x, y \in \mathscr{A}$. A positive linear functional $f$ on $\mathscr{A}$ is called $\Sigma$-regular (resp. regular) if $f^{\circ}$ is $\Sigma$-regular (resp. regular).

Let $\phi$ be a positive invariant sesquilinear form on $\mathscr{A} \times \mathscr{A}$. We denote

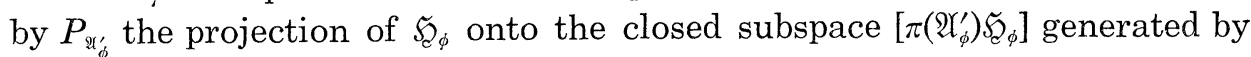
$\pi\left(\mathfrak{H}_{\phi}^{\prime}\right) \mathfrak{Y}_{\bar{\alpha}}$. We note that $P_{\mathscr{N}_{\phi}^{\prime}} \in \pi_{\phi}(\mathscr{A})^{\prime}$ if $\pi_{\phi}^{*}=\pi_{\phi}^{* *}$.

Proposition 5.2. Let $\phi$ be a positive invariant sesquilinear form on $\mathscr{A} \times \mathscr{A}$. Consider the following statements.

(1) $\phi$ is $\Sigma$-regular. 
(2) There exists a set $\left\{K_{\alpha}\right\}$ in $\mathfrak{Y}_{\phi}^{\prime}$ such that $\pi\left(K_{\alpha}\right) \geqq 0$ for each $\alpha$ and $\phi(x, y)$ $=\sum_{\alpha}\left(\pi_{\phi}^{*}(x) \lambda\left(K_{\alpha}\right) \mid \pi_{\phi}^{*}(y) \lambda\left(K_{\alpha}\right)\right)$ for each $x, y \in \mathscr{A}$.

(3) There exists a net $\left\{\phi_{\alpha}\right\}$ of strongly regular positive invariant sesquilinear forms on $\mathscr{A} \times \mathscr{A}$ such that $\phi_{a} \leqq \phi$ for each $\alpha$ and $\lim _{\alpha} \phi_{\alpha}(x, y)=\phi(x, y)$ for each $x, y \in \mathscr{A}$.

(4) $\phi$ is regular.

(5) There exists a net $\left\{K_{\alpha}\right\}$ in $\mathfrak{H}_{\phi}^{\prime}$ such that $0 \leqq \pi\left(K_{k}\right) \leqq I$ for each $\alpha$ and $\left\{\pi\left(K_{\alpha}\right)\right\}$ converges weakly to $I$.

(6) $P_{\mathfrak{x}_{\alpha}^{\prime}}=I$.

Then the following implications hold:

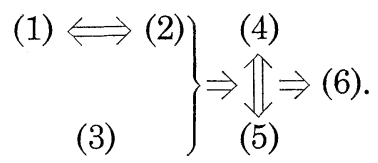

In particular, if $\pi_{\phi}^{*}=\pi_{\phi}^{* *}$, then the above statements (3) (6) are equivalent.

Proof. When $\pi_{\phi}^{*}=\pi_{\phi}^{* *}$, we show the implication $(6) \Rightarrow(3)$. The other implications follow immediately from Lemma 4.2. Since $P_{\mathscr{H}^{\prime}}=I$, it follows that $\pi\left(\mathfrak{U}_{\phi}^{\prime}\right)$ is a nondegenerate ${ }^{*}$-algebra on $\mathfrak{H}_{\phi}$, so that there exists a net $\left\{K_{\alpha}\right\}$ in $\mathfrak{Y}_{\dot{\phi}}^{\prime}$ such that $\left\|\pi\left(K_{\alpha}\right)\right\| \leqq 1$ for each $\alpha$ and $\left\{\pi\left(K_{\alpha}\right)\right\}$ converges strongly to $I$. We now put

$$
f_{\alpha}(x)=\left(\pi\left(K_{\alpha}\right) \lambda_{\dot{\phi}}(x) \mid \lambda\left(K_{\alpha}\right)\right)
$$

for $x \in \mathscr{A}$. Then we have

$$
\begin{aligned}
& f_{\alpha}\left(x^{*} x\right)=\left\|\pi\left(K_{\alpha}\right) \lambda_{\dot{\phi}}(x)\right\|^{2} \leqq \phi(x, x), \\
& \left|f_{\alpha}(x)\right|^{2} \leqq\left\|\lambda\left(K_{\alpha}\right)\right\|^{2}\left\|\pi\left(K_{\alpha}\right) \lambda_{\phi}(x)\right\|^{2}=\left\|\lambda\left(K_{\alpha}\right)\right\|^{2} f_{\alpha}\left(x^{*} x\right)
\end{aligned}
$$

for all $x \in \mathscr{A}$. Hence, $f_{\alpha}$ is a strongly regular positive linear functional on $\mathscr{A}$ with $f_{\alpha}^{\circ} \leqq \phi$. Furthermore, we have

$$
\begin{aligned}
\lim _{\alpha} f_{\alpha}^{\circ}(x, y) & =\lim _{\alpha}\left(\pi\left(K_{\alpha}\right) \lambda_{\dot{\phi}}(x) \mid \pi\left(K_{\alpha}\right) \lambda_{\dot{\phi}}(y)\right) \\
& =\left(\lambda_{\phi}(x) \mid \lambda_{\phi}(y)\right) \\
& =\phi(x, y)
\end{aligned}
$$

for all $x, y \in \mathscr{A}$. Hence, the statement (3) holds.

REMARK. We note by the equivalence of (3) and (4) in Proposition 5.2 that if for a positive invariant sesquilinear form $\phi$ on $\mathscr{A} \times \mathscr{A}$ there exists a net $\left\{\phi_{\alpha}\right\}$ of regular Riesz forms on $\mathscr{A}$ such that $\phi_{\alpha} \leqq \phi$ for each $\alpha$ and $\lim _{\alpha} \phi_{\alpha}(x, y)=\phi(x, y)$ for each $x, y \in \mathscr{A}$, then $\phi$ is regular.

We next investigate when a regular positive invariant sesquilinear form is $\Sigma$-regular. 
Let $\phi$ be a positive invariant sesquilinear form on $\mathscr{A} \times \mathscr{A}$. For each positive operator $K_{0}$ in $\pi_{\phi}(\mathscr{A})^{\prime}$ we put

$$
\phi_{K_{0}}(x, y)=\left(K_{0} \lambda_{\phi}(x) \mid \lambda_{\dot{\varphi}}(y)\right)
$$

for $x, y \in \mathscr{A}$. Then $\phi_{K_{0}}$ is a $\phi$-dominated positive invariant sesquilinear form on $\mathscr{A} \times \mathscr{A}$. By Lemma 4.2 we see that every $\phi$-dominated positive invariant sesquilinear form is of this form.

THEOREM 5.3. Suppose that $\phi$ is a positive invariant sesquilinear form on $\mathscr{A} \times \mathscr{A}$ satisfying $\pi_{\phi}^{*}=\pi_{\phi}^{* *}$. Consider the following statements.

(1) $\phi_{E}$ is $\Sigma$-regular for each non-zero projection $E$ in $\pi_{\phi}(\mathscr{A})^{\prime}$.

(2) $\phi_{E}$ is regular for each non-zero projection $E$ in $\pi_{\phi}(\mathscr{A})^{\prime}$.

(3) There exists a set $\left\{E_{\alpha}\right\}$ in $\mathfrak{Y}_{\phi}^{\prime}$ such that $\left\{\pi\left(E_{\alpha}\right)\right\}$ is of mutually orthogonal projections and $\sum_{\alpha} \pi\left(E_{\alpha}\right)=I$.

(4) There exists a set $\left\{\xi_{\alpha}\right\}$ in $\mathscr{D}\left(\pi_{\phi}^{*}\right)$ such that

(4.1) $\left(\pi_{\phi}^{*}(x) \xi_{\alpha} \mid \pi_{\phi}^{*}(y) \xi_{\beta}\right)=0$ for each $x, y \in \mathscr{A}$ and $\alpha \neq \beta$;

(4.2) $\lambda_{\dot{\phi}}(x)=\sum_{\alpha} \pi_{\phi}^{*}(x) \xi_{\alpha}$ for each $x \in \mathscr{A}$.

(5) There exists a set $\left\{\xi_{\alpha}\right\}$ in $\mathscr{D}\left(\pi_{\phi}^{*}\right)$ such that

(5.1) $\left(\pi_{\phi}^{*}(x) \xi_{\alpha} \mid \pi_{\phi}^{*}(y) \xi_{\beta}\right)=0$ for each $x, y \in \mathscr{A}$ and $\alpha \neq \beta$;

(5.2) $\sum_{\alpha}\left\|\pi_{\phi}^{*}(x) \xi_{\alpha}\right\|^{2}<\infty$ for each $x \in \mathscr{A}$;

(5.3) $\left\{\sum_{\alpha} \pi_{\phi}^{*}(x) \xi_{\alpha} ; x \in \mathscr{A}\right\}$ is dense in $\mathfrak{S}_{\phi}$;

(5.4) $\phi(x, y)=\sum_{\alpha}\left(\pi_{\phi}^{*}(x) \xi_{\alpha} \mid \pi_{\phi}^{*}(y) \xi_{\alpha}\right)$ for each $x, y \in \mathscr{A}$.

(6) There exists a set $\left\{\xi_{\alpha}\right\}$ in $\mathscr{D}\left(\pi_{\phi}^{*}\right)$ such that

(6.1) $\left(\pi_{\phi}^{*}(x) \xi_{\phi} \mid \pi_{\phi}^{*}(y) \xi_{\beta}\right)=0$ for each $x, y \in \mathscr{A}$ and $\alpha \neq \beta$;

(6.2) $\phi(x, y)=\sum_{\alpha}\left(\pi_{\phi}^{*}(x) \xi_{\alpha} \mid \pi_{\phi}^{*}(y) \xi_{\alpha}\right)$ for each $x, y \in \mathscr{A}$.

(7) $\phi$ is $\Sigma$-regular.

Then the following implications hold;

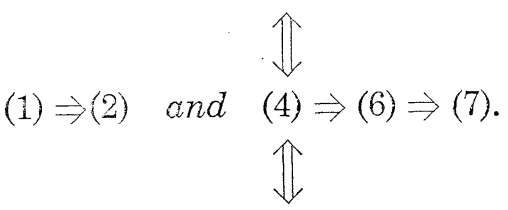

(5)

In particular, if $\dot{\phi}$ is a Riesz form on $\mathscr{A}$, then the implication $(2) \Rightarrow(3)$ holds.

Proof. $\quad(1) \Rightarrow(2)$ This follows since the $\Sigma$-regularity implies the regularity.

(3) $\Rightarrow(4) \Rightarrow(5) \quad$ This is trivial.

(5) $\Rightarrow$ (3) We put 


$$
U_{0} \lambda_{\phi}(x)=\sum_{\alpha} \pi_{\varphi}^{*}(x) \xi_{\alpha}
$$

for $x \in \mathscr{A}$. Then $U_{0}$ is extended to a unitary operator $U$ on $\tilde{\mathfrak{S}}_{\phi}$ which is contained in $\pi_{\phi}(\mathscr{A})^{\prime}$. Since $\pi_{\phi}^{*}$ is a self-adjoint representation of $\mathscr{A}$, we have

$$
\lambda_{\phi}(x)=\sum_{\alpha} \pi_{\phi}^{*}(x) U^{*} \xi_{\alpha}
$$

for each $x \in \mathscr{A}$. We now denote by $E_{\alpha}^{0}$ the projection of $\mathfrak{S}_{\phi}$ onto $\left[\pi_{\phi}^{*}(\mathscr{A}) U^{*} \xi_{\alpha}\right]$. Then it follows since $U$ is a unitary operator that $\left\{E_{\alpha}^{0}\right\}$ is a set of mutually orthogonal projections in $\pi_{\phi}(\mathscr{A})^{\prime},\left(E_{\alpha}^{0}, U^{*} \xi_{\alpha},\left(U^{*} \xi_{\alpha}\right)^{*}\right) \in \mathfrak{Y}_{\dot{\phi}}^{\prime}$ for each $\alpha$ and $\sum_{\alpha} E_{\alpha}^{0}=I$.

$(5) \Rightarrow(6) \quad$ This is trivial.

$(6) \Rightarrow(7) \quad$ We put

$$
f_{\alpha}(x)=\left(\pi_{\phi}^{*}(x) \xi_{\alpha} \mid \xi_{\alpha}\right)
$$

for $x \in \mathscr{A}$. Since $\pi_{\phi}^{*}$ is a $*$-representation of $\mathscr{A}$, we have

$$
\begin{aligned}
& f_{\alpha}\left(x^{*} x\right)=\left\|\pi_{\phi}^{*}(x) \xi_{\alpha}\right\|^{2} \leqq \sum_{\alpha}\left\|\pi_{\phi}^{*}(x) \xi_{\alpha}\right\|^{2}=\phi(x, x), \\
& \left|f_{\alpha}(x)\right|^{2} \leqq\left\|\xi_{\alpha}\right\|^{2}\left\|\pi_{\phi}^{*}(x) \xi_{\alpha}\right\|^{2} \leqq\left\|\xi_{\alpha}\right\|^{2} \phi(x, x)
\end{aligned}
$$

for all $x \in \mathscr{A}$, and hence $\left\{f_{\alpha}\right\}$ is a set of $\phi$-majorized positive linear functionals on $\mathscr{A}$ and $\sum_{\alpha} f_{\alpha}^{\circ}(x, y)=\phi(x, y)$ for all $x, y \in \mathscr{A}$, which means that $\phi$ is $\Sigma$-regular.

$(2) \Rightarrow(3)$ Suppose that $\phi$ is a Riesz form on $\mathscr{A}$. Let $\left\{E_{\lambda}\right\}_{\lambda \in A}$ be a maximal family in $\mathfrak{Y}_{\phi}^{\prime}$ satisfying that $\left\{\pi\left(E_{\lambda}\right)\right\}_{\lambda \in A}$ is of mutually orthogonal projections. We show $\sum_{\lambda \in A} \pi\left(E_{\lambda}\right)=I$. Suppose that $E_{\Lambda} \equiv I-\sum_{\lambda \in A} \pi\left(E_{\lambda}\right) \neq 0$. Then $\phi_{E_{A}}$ is a non-zero positive invariant sesquilinear form on $\mathscr{A} \times \mathscr{A}$. Since $\phi_{E_{A}}$ is regular by the assumption, there exists a $\phi_{E_{A}}$-majorized positive linear functional $f$ on $\mathscr{A}$ with $f^{\circ} \neq 0$. Since $\phi_{E_{A}} \leqq \phi, f$ is also $\phi$-majorized. Hence, by Lemma 4.2 there exists an element $K$ of $\mathfrak{Y}_{\phi}^{\prime}$ such that $\pi(K) \geqq 0$ and $f(x)=\left(\lambda_{\phi}(x) \mid \lambda(K)\right)$ for all $x \in \mathscr{A}$. Since $f$ is $\phi_{E_{A}}$-majorized, there is a constant $\gamma$ such that $|f(x)|^{2} \leqq$ $\gamma \phi_{E_{A}}(x, x)$ for all $x \in \mathscr{A}$. Hence, we have

$$
\begin{aligned}
\left|\left(\lambda_{\phi}(y) \mid \pi(K) \lambda_{\phi}(x)\right)\right|^{2} & =\left|f\left(x^{*} y\right)\right|^{2} \leqq \gamma \phi_{E_{A}}\left(x^{*} y, x^{*} y\right) \\
& =\gamma\left(\pi_{\phi}\left(x^{*}\right) E_{\Lambda} \lambda_{\phi}(y) \mid \pi_{\phi}\left(x^{*}\right) \lambda_{\phi}(y)\right)
\end{aligned}
$$

for each $x, y \in \mathscr{A}$. Since $\phi$ is a Riesz form on $\mathscr{A}$, it follows that

$$
\left|\left(\xi \mid \pi(K) \lambda_{\dot{\phi}}(x)\right)\right|^{2} \leqq \gamma\left(\pi_{\phi}\left(x^{*}\right) E_{A} \xi \mid \pi_{\phi}\left(x^{*}\right) \xi\right)
$$

for each $x \in \mathscr{A}$ and $\xi \in \mathscr{D}\left(\pi_{\phi}\right)$, which implies that $\left(\left(I-E_{A}\right) \lambda_{\phi}(y) \mid \pi(K) \lambda_{\phi}(x)\right)=0$ for each $x, y \in \mathscr{A}$. Hence, $\pi(K) E_{A}=\pi(K)$. Since $f^{\circ} \neq 0$, we have $\pi(K) \neq 0$. Let $\pi(K)=\int_{0}^{\|\pi(K)\|} \lambda d E(\lambda)$ be the spectral resolution of $\pi(K)$. We now put 


$$
E_{t}^{\circ}=\int_{t}^{\|\pi(K)\|} d E(\lambda) \text { and } X_{t}=\int_{t}^{\|\pi(K)\|} \frac{1}{\lambda} d E(\lambda)
$$

for $0<t<\|\pi(K)\|$. Then, it follows that $E_{t}^{\circ}, X_{t} \in \pi_{\phi}(\mathscr{A})^{\prime}$ and $E_{t}^{\circ}=\pi(K) X_{t}^{2} \pi(K)$. Since $\pi(K) \neq 0$, we have $E_{t_{0}}^{\circ} 0$ for some $t_{0} \in(0,\|\pi(K)\|)$. Furthermore, we have

$$
\begin{aligned}
E_{t_{0}}^{\circ} \lambda_{\phi}(x) & =\pi(K) X_{t_{0}}^{2} \pi(K) \lambda_{\phi}(x) \\
& =\pi(K) X_{t_{0}}^{2} \pi_{\phi}(x) \lambda(K) \\
& =\pi_{\phi}(x) \pi(K) X_{t_{0}}^{2} \lambda(K)
\end{aligned}
$$

for all $x \in \mathscr{A}$. It hence follows that $E_{t_{0}}=\left(E_{t_{0}}^{\circ}, \pi(K) X_{t_{0}}^{2} \lambda(K),\left(\pi(K) X_{t_{0}}^{2} \lambda(K)\right)^{*}\right)$ $\in \mathfrak{Y}_{\phi}^{\prime}$ and $E_{t_{0}}^{\circ} E_{A}=\pi(K) X_{t_{0}}^{2} \pi(K) E_{A}=\pi(K) X_{t_{0}}^{2} \pi(K)=E_{t_{0}}^{\circ}$, which contradicts the maximality of $\left\{E_{\lambda}\right\}_{\lambda \in A}$. Hence, $\sum_{\lambda \in A} \pi\left(E_{\lambda}\right)=I$. This completes the proof.

We next investigate when a positive invariant sesquilinear form $\psi$ on $\mathscr{A} \times \mathscr{A}$ with $\psi \leqq \phi$ is $\Sigma$-regular (resp. regular), where $\phi$ is a $\Sigma$-regular (resp. regular) positive invariant sesquilinear form on $\mathscr{A} \times \mathscr{A}$.

LEMMA 5.4. Let $\phi$ be a positive invariant sesquilinear form on $\mathscr{A} \times \mathscr{A}$ satisfying $\pi_{\phi}^{*}=\pi_{\phi}^{* *}$. Suppose that $\phi$ is regular (resp. $\sum$-regular); that is, there exists a set $\left\{K_{\alpha}\right\}$ in $\mathfrak{Y}_{\phi}^{\prime}$ such that $\phi(x, y)=\lim _{\alpha}\left(\pi_{\phi}^{*}(x) \lambda\left(K_{\alpha}\right) \mid \pi_{\phi}^{*}(y) \lambda\left(K_{\alpha}\right)\right)$ (resp. $\left.\phi(x, y)=\sum_{\alpha}\left(\pi_{\phi}^{*}(x) \lambda\left(K_{\alpha}\right) \mid \pi_{\phi}^{*}(y) \lambda\left(K_{\alpha}\right)\right)\right)$ for each $x, y \in \mathscr{A}$. Then the following statements hold.

(1) If $E$ is an element of $\mathfrak{N}_{\phi}^{\prime}$ such that $\pi(E)$ is a projection, then $\phi_{\pi(E)}$ is regular (resp. $\Sigma$-regular).

(2) If $K_{0}$ is a positive operator in $\pi_{\phi}(\mathscr{A})^{\prime}$ such that $K_{0} \pi\left(K_{\alpha}\right)=\pi\left(K_{\alpha}\right) K_{0}$ for each $\alpha$, then $\phi_{K_{0}}$ is regular (resp. $\Sigma$-regular).

Proof. Suppose that $\phi$ is regular.

(1) We now put

$$
f_{\alpha}(x)=\left(\pi\left(K_{\alpha}\right) * \pi\left(K_{\alpha}\right) \pi(E) \lambda_{\phi}(x) \mid \lambda(E)\right)
$$

for $x \in \mathscr{A}$. Then it follows that $f_{\alpha}$ is a $\phi_{\pi(E)}$-majorized positive linear functional on $\mathscr{A}$ satisfying that $f_{\alpha}^{\circ} \leqq \phi_{\pi(E)}$ and $\lim _{\alpha} f_{\alpha}^{\circ}(x, y)=\phi_{\pi(E)}(x, y)$ for each $x, y \in \mathscr{A}$. Hence, $\phi_{\pi(E)}$ is regular.

(2) A set $\left\{f_{\alpha}\right\}$ of positive linear functionals on $\mathscr{A}$ defined by

$$
f_{\alpha}(x)=\left(K_{0} \pi\left(K_{\alpha}\right) \lambda_{\phi}(x) \mid \lambda\left(K_{\alpha}\right)\right)
$$

for $x \in \mathscr{A}$ implies our assertion.

We can similarly show that $\phi_{\pi(E)}$ and $\phi_{K_{0}}$ are $\Sigma$-regular if $\phi$ is $\Sigma$-regular. This completes the proof.

LEMMA 5.5. If $\dot{\phi}$ is a $\Sigma$-regular (resp. regular) Riesz form on a commuta- 
tive $*$-algebra $\mathscr{A}$, then every positive invariant sesquilinear form $\psi$ on $\mathscr{A} \times \mathscr{A}$ with $\psi \leqq \phi$ is $\Sigma$-regular (resp. regular).

Proof. Suppose that $\phi$ is regular. By Proposition 5.2, it follows that $\pi\left(\mathfrak{U}_{\phi}^{\prime}\right)$ is a nondegenerate ${ }^{*}$-algebra on $\mathfrak{S}_{\phi}$, so that there exists a set $\left\{K_{\alpha}\right\}$ in $\mathfrak{A}_{\phi}^{\prime}$ such that $\left\|\pi\left(K_{\alpha}\right)\right\| \leqq 1$ for each $\alpha$ and $\left\{\pi\left(K_{\alpha}\right)\right\}$ converges strongly to $I$. Since

$$
\begin{aligned}
\left(\lambda_{\phi}\left(x^{*}\right) \mid \lambda_{\phi}\left(y^{*}\right)\right) & =\lim _{\alpha}\left(\pi\left(K_{\alpha}\right) \lambda_{\phi}\left(x^{*}\right) \mid \lambda_{\phi}\left(y^{*}\right)\right) \\
& =\lim _{\alpha}\left(\pi_{\phi}\left(x^{*}\right) \lambda\left(K_{\alpha}\right) \mid \lambda_{\alpha}\left(y^{*}\right)\right) \\
& =\lim _{\alpha}\left(\lambda\left(K_{\alpha}\right) \mid \lambda_{\phi}\left(x y^{*}\right)\right) \\
& =\lim _{\alpha}\left(\lambda\left(K_{\alpha}\right) \mid \lambda_{\phi}\left(y^{*} x\right)\right) \\
& =\lim _{\alpha}\left(\pi\left(K_{\alpha}\right) \lambda_{\phi}(y) \mid \lambda_{\phi}(x)\right) \\
& =\left(\lambda_{\phi}(y) \mid \lambda_{\phi}(x)\right)
\end{aligned}
$$

for each $x, y \in \mathscr{A}$, the map $\lambda_{\phi}(x) \rightarrow \lambda_{\phi}\left(x^{*}\right)$ is extended to a reflexive conjugate linear isometry $J_{\phi}$ on $\mathfrak{S}_{\phi}$. Furthermore, we have

$$
\begin{aligned}
\left(J_{\phi} \lambda(K) \mid \lambda_{\phi}(x y)\right) & =\left(\lambda_{\phi}\left(y^{*} x^{*}\right) \mid \lambda(K)\right) \\
& =\left(\pi(K)^{*} \lambda_{\phi}\left(x^{*}\right) \mid \lambda_{\dot{\phi}}(y)\right) \\
& =\left(\lambda\left(K^{+}\right) \mid \lambda_{\phi}(x y)\right)
\end{aligned}
$$

for each $x, y \in \mathscr{A}$ and $K \in \mathfrak{U}_{\phi}^{\prime}$. Since $\left[\pi\left(\mathfrak{U}_{\phi}^{\prime}\right) \lambda_{\phi}(\mathscr{A})\right]=\left[\pi_{\phi}(\mathscr{A}) \lambda\left(\mathfrak{U}_{\phi}^{\prime}\right)\right] \subset\left[\pi_{\phi}(\mathscr{A}) \mathscr{D}\left(\pi_{\phi}\right)\right]$ $=\left[\lambda_{\phi}\left(\mathscr{A}^{2}\right)\right]$, we have $J_{\phi} \lambda(K)=\lambda\left(K^{+}\right)$for each $K \in \mathfrak{A}_{\phi}^{\prime}$. Take arbitrary $C \in \pi_{\phi}(\mathscr{A})^{\prime}$ and $K \in \mathfrak{U}_{\dot{\phi}}^{\prime}$. Since $\lambda(K) \in \mathscr{D}\left(\pi_{\dot{\phi}}\right)$ by the self-adjointness of $\pi_{\phi}$, there exists a net $\left\{x_{\beta}\right\}$ in $\mathscr{A}$ such that $\left\{\lambda_{\phi}\left(x_{\beta}\right)\right\}$ converges to $\lambda(K)$ with respect to the $t_{\pi_{\phi}(\mathscr{A})^{-}}$topology. Then, by the above argument we have

$$
\lim _{\beta} \lambda_{\phi}\left(x_{\beta}^{*}\right)=\lim _{\beta} J_{\phi} \lambda_{\phi}\left(x_{\beta}\right)=J_{\phi} \lambda(K)=\lambda\left(K^{+}\right) .
$$

Since

$$
\begin{aligned}
\left(C \pi(K) \lambda_{\phi}(x) \mid \lambda_{\dot{\phi}}(y)\right) & =\lim _{\beta}\left(C \pi_{\phi}(x) \lambda_{\phi}\left(x_{\beta}\right) \mid \lambda_{\phi}(y)\right) \\
& =\lim _{\beta}\left(C \lambda_{\phi}(x) \mid \pi_{\phi}(y) \lambda_{\phi}\left(x_{\beta}^{*}\right)\right) \\
& =\left(\pi_{\phi}(y)^{*} C \lambda_{\phi}(x) \mid \lambda\left(K^{+}\right)\right) \\
& =\left(\pi(K) C \lambda_{\phi}(x) \mid \lambda_{\phi}(y)\right)
\end{aligned}
$$

for each $x, y \in \mathscr{A}$, we have $C \pi(K)=\pi(K) C$. It hence follows from Lemma 5.4 that a positive invariant sesquilinear form $\psi$ on $\mathscr{A} \times \mathscr{A}$ with $\psi \leqq \phi$ is regular (resp. $\Sigma$-regular) if $\phi$ is regular (resp. $\Sigma$-regular). 
TheORem 5.6. Let $\phi$ be a Riesz form on a commutative *algebra $\mathscr{A}$. Consider the following statements.

(1) $\phi$ is regular.

(2) $\phi$ is $\Sigma$-regular.

(3) There exists a net $\left\{\xi_{\alpha}\right\}$ in $\mathscr{D}\left(\pi_{\dot{\phi}}\right)$ such that

(3.1) $\left(\pi_{\phi}(x) \xi_{\alpha} \mid \pi_{\phi}(y) \xi_{\beta}\right)=0$ for each $x, y \in \mathscr{A}$ and $\alpha \neq \beta$;

(3.2) $\quad \sum_{\alpha}\left\|\pi_{\phi}(x) \xi_{\alpha}\right\|^{2}<\infty$ for each $x \in \mathscr{A}$;

(3.3) $\left\{\sum \pi_{\dot{\phi}}(x) \xi_{\alpha} ; x \in \mathscr{A}\right\}$ is dense in $\mathfrak{S}_{\phi}$;

(3.4) $\phi(x, y)=\sum_{\alpha}\left(\pi_{\phi}(x) \xi_{\alpha} \mid \pi_{\phi}(y) \xi_{\alpha}\right)$ for each $x, y \in \mathscr{A}$.

(4) $\phi$ is nondegenerate; that is, $\lambda_{\phi}\left(\mathscr{A}^{2}\right)$ is total in $\mathscr{S}_{\phi}$.

(5) $\pi_{\phi}(\mathscr{A})^{\prime}$ is an abelian von Neumann algebra.

Then the following implications hold:

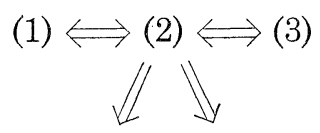

(4) (5).

In particular, if $\pi_{\phi}$ is standard; that is, $\pi_{\dot{\phi}}(x)^{*}=\overline{\pi_{\phi}\left(x^{*}\right)}$ for each $x \in \mathscr{A}$, then the statements $(1) \sim(4)$ are equivalent. In this case, $\pi_{\phi}(\mathscr{A})^{\prime}$ is a maximal abelian von Neumann algebra.

Proof. The equivalence of (1) (3) follows from Theorem 5.3 and Lemma 5.5.

$(3) \Rightarrow(4) \quad$ This is proved in the proof of Lemma 5.5.

$(1) \Rightarrow(5)$ Since $\phi$ is regular, there exists a net $\left\{K_{\alpha}\right\}$ in $\mathfrak{H}_{\phi}^{\prime}$ such that $\left\|\pi\left(K_{\alpha}\right)\right\| \leqq 1$ for each $\alpha$ and $\left\{\pi\left(K_{\alpha}\right)\right\}$ converges strongly to $I$. In the proof of Lemma 5.5 we have seen that $\pi(K) C=C \pi(K)$ for each $K \in \mathfrak{Y}_{\phi}^{\prime}$ and $C \in \pi_{\phi}(\mathscr{A})^{\prime}$. Furthermore, we note that $\pi(S) C \pi(K) \in \mathfrak{U}_{\dot{\phi}}^{\prime}$ for each $S, K \in \mathfrak{Y}_{\phi}^{\prime}$ and $C \in \pi_{\dot{\phi}}(\mathscr{A})^{\prime}$. Hence, we have

$$
\begin{aligned}
\left(C_{1} C_{2} \lambda_{\phi}(x) \mid \lambda_{\phi}(y)\right) & =\lim _{\alpha}\left(\pi\left(K_{\alpha}\right) * C_{1} \pi\left(K_{\alpha}\right) C_{2} \lambda_{\phi}(x) \mid \lambda_{\phi}(y)\right) \\
& =\lim _{\alpha}\left(C_{2} \pi\left(K_{\alpha}\right) * C_{1} \pi\left(K_{\alpha}\right) \lambda_{\phi}(x) \mid \lambda_{\dot{\phi}}(y)\right) \\
& =\left(C_{2} C_{1} \lambda_{\phi}(x) \mid \lambda_{\dot{\phi}}(y)\right)
\end{aligned}
$$

for each $C_{1}, C_{2} \in \pi_{\phi}(\mathscr{A})^{\prime}$ and $x, y \in \mathscr{A}$, and so $C_{1} C_{2}=C_{2} C_{1}$. Hence, $\pi_{\phi}(\mathscr{A})^{\prime}$ is an abelian von Neumann algebra.

$(2) \Rightarrow(3)$ Suppose that $\pi_{\phi}$ is standard and $\phi$ is non-degenerate. Take arbitrary $x \in \mathscr{A}$. Let $\overline{\pi_{\phi}(x)}=U \overline{\mid \pi_{\phi}(x)} \mid$ be the polar decomposition and $\overline{\left|\pi_{\phi}(x)\right|}$ $=\int_{0}^{\infty} \lambda d E(\lambda)$ be the spectral resolution of $\left|\overline{\pi_{\phi}(x)}\right|$. We now put

$$
X_{n}=\int_{-n}^{n} \lambda d E(\lambda) \text { and } E_{n}=\int_{-n}^{n} d E(\lambda)
$$


for $n=1,2, \cdots$. Since $\overline{\pi_{\phi}(x)}$ is affiliated with $\pi_{\phi}(\mathscr{A})^{\prime \prime}$ and $\pi_{\phi}(\mathscr{A})^{\prime \prime} \subset \pi_{\phi}(\mathscr{A})^{\prime}$ ([22] Theorem 7.1), we have $U, E_{n}, X_{n} \in \pi_{\dot{\phi}}(\mathscr{A})^{\prime \prime} \cap \pi_{\dot{\phi}}(\mathscr{A})^{\prime}$ for $n=1,2, \cdots$, which implies that $\left(U X_{n}, U E_{n} \lambda_{\phi}(x),\left(U E_{n} \lambda_{\phi}\left(x^{*}\right)\right)^{*}\right) \in \mathfrak{Y}_{\phi}^{\prime}$ and $\lim U X_{n} \lambda_{\phi}(y)=\lambda_{\phi}(x y)$ for each $y \in \mathscr{A}$. Hence, it follows since $\lambda_{\phi}\left(\mathscr{A}^{2}\right)$ is total in ${ }^{n \rightarrow \infty} \tilde{\mathfrak{C}}_{\phi}$ that $\phi$ is regular, so that $\pi_{\phi}(\mathscr{A})^{\prime}$ is maximal abelian by $([22]$ Theorem 7.1) and $(3) \Rightarrow(5)$. This completes the proof.

EXAMPLES. (1) Every admissible continuous positive invariant sesquilinear form on a commutative locally convex *-algebra with an approximate identity is $\Sigma$-regular.

(2) Let $\mathscr{A}$ be a locally convex $*$-algebra with an approximate identity $\left\{e_{\alpha}\right\}$. Every continuous positive invariant sesquilinear form $\phi$ on $\mathscr{A} \times \mathscr{A}$ satisfying that $\phi\left(x e_{\alpha}, x e_{\alpha}\right) \leqq \phi(x, x)$ for each $\alpha$ and $x \in \mathscr{A}$ is regular.

(3) Let $\mathscr{A}$ be a $*$-algebra and $\phi$ be a positive invariant sesquilinear form on $\mathscr{A} \times \mathscr{A}$. If there exists a net $\left\{\phi_{\alpha}\right\}$ of admissible positive invariant sesquilinear forms on $\mathscr{A} \times \mathscr{A}$ such that $\phi_{\alpha} \leqq \phi$ for each $\alpha$ and $\lim _{\alpha} \phi_{\alpha}(x, y)=$ $\phi(x, y)$ for all $x, y \in \mathscr{A}$, then $\phi$ is called approximately admissible. Suppose that $\phi$ is an approximately admissible positive invariant sesquilinear form on $\mathscr{A} \times \mathscr{A}$ satisfying $\pi_{\phi}^{*}=\pi_{\phi}^{* *}$. Then we see that there exists a set $\left\{E_{\lambda}\right\}_{\lambda \in A}$ of mutually orthogonal projections in $\pi_{\phi}(\mathscr{A})^{\prime}$ such that $\sum_{\lambda \in A} E_{\lambda}=I$ and $\overline{\pi_{\phi}^{*}(x) E_{\lambda}}$ is bounded for each $x \in \mathscr{A}$ and $\lambda \in A$. A locally convex topology on the closed $O_{p}^{*}$-algebra $\pi_{\phi}(\mathscr{A})$ generated by a family of seminorms $\left\{\|\|_{\lambda} ; \lambda \in \Lambda\right\}$, where $\left\|\pi_{\phi}(x)\right\|_{\lambda}$ denotes the uniform norm $\| \overline{\pi_{\phi}^{*}(x) E_{\lambda} \|}$ of $\overline{\pi_{\phi}^{*}(x) E_{\lambda}}$, is said to be a locally uniform topology $t_{t u}$. If the map $\pi_{\phi}(x) \in\left(\pi_{\phi}(\mathscr{A}), t_{l u}\right) \rightarrow \lambda_{\phi}(x) \in \mathfrak{S}_{\phi}$ is continuous, then $\phi$ is $\Sigma$-regular. In fact, since $\pi_{\phi}(x) \rightarrow \lambda_{\dot{\phi}}(x)$ is locally uniformly continuous, there exist a constant $\gamma$ and a finite subset $\left\{\lambda_{1}, \cdots, \lambda_{n}\right\}$ of $\Lambda$ such

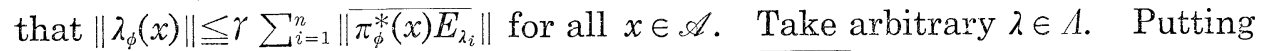
$E=\sum_{i=1}^{n} E_{\lambda_{i}}+E_{\lambda}$, we have $\left\|E \lambda_{\phi}(x)\right\| \leqq(n+1) \gamma \| \overline{\pi_{\phi}^{*}(x) E \|}$ for all $x \in \mathscr{A}$. Similarly to the proof $(5) \Rightarrow(2)$ of Theorem 4.4 , we can show that there exists an element $\xi$ of $E S_{S_{\phi}}$ such that $E \lambda_{\phi}(x)=\pi_{\phi}^{*}(x) \xi$ for all $x \in \mathscr{A}$. Hence, it follows since $\xi_{\lambda}=$ $E_{\lambda} \xi \in E_{\lambda} \mathfrak{Y}_{\phi}$ and $E_{\lambda} \lambda_{\phi}(x)=\pi_{\phi}^{*}(x) \xi_{\lambda}$ for all $x \in \mathscr{A}$ that $\lambda_{\phi}(x)=\sum_{\lambda \in \Lambda} \pi_{\phi}^{*}(x) \xi_{\lambda}$ for all $x \in \mathscr{A}$, which implies that $\phi$ is $\Sigma$-regular.

(4) Let $\mathscr{M}$ be a self-adjoint $O_{p}^{*}$-algebra on a dense subspace $\mathscr{D}$ in a Hilbert space $\mathfrak{S}_{2}$. A linear functional $f$ on $\mathscr{M}$ is called weakly continuous (resp. $\sigma$-weakly continuous) if $|f(x)| \leqq\left|\sum_{i=1}^{n}\left(x \xi_{i} \mid \eta_{i}\right)\right|\left(\right.$ resp. $\left.|f(x)| \leqq\left|\sum_{i=1}^{\infty}\left(x \xi_{i} \mid \eta_{i}\right)\right|\right)$ for all $x \in \mathscr{A}$, where $\left\{\xi_{i}\right\},\left\{\eta_{i}\right\} \subset \mathscr{D}_{\infty}(\mathscr{M})=\left\{\left\{\xi_{i}\right\} \subset \mathscr{D} ; \sum_{i=1}^{\infty}\left\|\xi_{i}\right\|^{2}<\infty\right.$ and $\sum_{i=1}^{\infty}\left\|x \xi_{i}\right\|^{2}$ $<\infty$ for each $x \in \mathscr{M}\}$. Then we see that every weakly continuous (resp. $\sigma$-weakly continuous) linear functional on $\mathscr{M}$ is a linear combination of strongly regular (resp. $\Sigma$-regular) positive linear functionals on $\mathscr{M}$.

(5) Let $\phi$ be a weight on a $C^{*}$-algebra $\mathscr{A}$. Then a positive invariant sesquilinear form $\phi^{\circ}$ on $\mathfrak{N}_{\phi} \times \mathfrak{N}_{\dot{\phi}}$ is defined by $\phi^{\circ}(x, y)=\phi\left(y^{*} x\right)$ for $x, y \in \mathfrak{N}_{\phi}$, 
where $\mathfrak{N}_{\phi}=\left\{x \in \mathscr{A} ; \phi\left(x^{*} x\right)<\infty\right\}$. With the aid of Combes [4] and Pedersen, Takesaki [21] we shall show in Section 7 that $\phi$ is uniformly lower semicontinuous on $\mathscr{D}(\phi)=\{x \in \mathscr{A} ; x \geqq 0, \phi(x)<\infty\}$ if and only if $\phi^{\circ}$ is regular if and only if $\phi^{\circ}$ is $\Sigma$-regular. In particular, if $\phi$ is a positive $C^{*}$-integral on a $C^{*}$-algebra, then $\phi^{\circ}$ is $\Sigma$-regular [20]. We also see by Haagerup [11] that if $\phi$ is a normal weight on a von Neumann algebra, then $\phi^{\circ}$ is $\Sigma$-regular.

\section{§6. Decomposition of positive invariant sesquilinear forms.}

In this section we show that every positive invariant sesquilinear form $\phi$ satisfying $\pi_{\phi}^{*}=\pi_{\phi}^{* *}$ is decomposed into the sum of the regular part and the singular part. Throughout this section let $\mathscr{A}$ be a $*$-algebra without identity.

We first introduce the notion of singularity of positive invariant sesquilinear forms.

DEFINITION 6.1. Let $\phi$ be a positive invariant sesquilinear form on $\mathscr{A} \times \mathscr{A}$. If there does not exist any $\phi$-majorized positive linear functional $f$ such that $f^{\circ} \neq 0$, then $\phi$ is called singular. If $\phi(x y, z)=0$ for each $x, y, z \in \mathscr{A}$; that is, $\pi_{\phi}(\mathscr{A})=\{0\}$, then $\phi$ is called nilpotent. A positive linear functional $f$ is called singular (resp. nilpotent) if $f^{\circ}$ is singular (resp. nilpotent).

Proposition 6.2. Let $\phi$ be a positive invariant sesquilinear form on $\mathscr{A} \times \mathscr{A}$. Consider the following statements.

(1) $\phi$ is nilpotent.

(2) $\pi_{\phi}^{*}(x) \in\left(\pi_{\phi}^{*}(\mathscr{A}), t_{w}\right) \rightarrow \lambda_{\phi}(x) \in \mathfrak{S}_{\phi}$ is singular, where the weak topology $t_{w}$ on $\pi_{\phi}^{*}(\mathscr{A})$ is a locally convex topology generated by the seminorms: $P_{\xi, \eta}\left(\pi_{\phi}^{*}(x)\right.$ ) $=\left(\pi_{\phi}^{*}(x) \xi \mid \eta\right)$ for $\xi, \eta \in \mathscr{D}\left(\pi_{\phi}^{*}\right)$.

(3) $P_{2 \phi^{\prime}}=0$.

(4) $\phi$ is singular.

Then the following implications hold:

$$
(1) \Rightarrow(2) \Rightarrow(3) \Rightarrow(4) \text {. }
$$

In particular, if $\pi_{\phi}^{*}=\pi_{\phi}^{* *}$, then the statements (3) and (4) are equivalent.

Proof. $\quad(1) \Rightarrow(2) \quad$ This is trivial.

$(2) \Rightarrow(3)$ Since $\pi_{\phi}^{*}(x) \rightarrow \lambda_{\phi}(x)$ is singular, for each $x \in \mathscr{A}$ there exists a net $\left\{x_{\alpha}\right\}$ in $\mathscr{A}$ such that $\lim _{\alpha}\left(\pi_{\phi}^{*}\left(x_{\alpha}\right) \xi \mid \eta\right)=0$ for each $\xi, \eta \in \mathscr{D}\left(\pi_{\phi}^{*}\right)$ and $\lim _{\alpha} \lambda_{\phi}\left(x_{\alpha}\right)=$ $\lambda_{\phi}(x)$. Hence, we have

$$
\begin{aligned}
\left(\pi(K) \lambda_{\phi}(x) \mid \lambda_{\phi}(y)\right) & =\lim _{\alpha}\left(\pi(K) \lambda_{\phi}\left(x_{\alpha}\right) \mid \lambda_{\phi}(y)\right) \\
& =\lim _{\alpha}\left(\pi_{\phi}^{*}\left(x_{\alpha}\right) \lambda(K) \mid \lambda_{\phi}(y)\right) \\
& =0
\end{aligned}
$$


for each $K \in \mathfrak{Y}_{\phi}^{\prime}$ and $x, y \in \mathscr{A}$, which implies that $P_{\mathfrak{l} \phi}^{\prime}=0$.

$(3) \Rightarrow(4) \quad$ This follows from Lemma 4.2 .

(4) $\Rightarrow(3)$ Suppose $\pi_{\phi}^{*}=\pi_{\phi}^{* *}$. Take arbitrary $K \in \mathfrak{Y}_{\dot{\phi}}^{\prime}$. We put

$$
f(x)=\left(\pi_{\phi}^{*}(x) \lambda(K) \mid \lambda(K)\right)
$$

for $x \in \mathscr{A}$. Then it follows from $\pi_{\phi}^{*}=\pi_{\phi}^{* *}$ that $f$ is a $\phi$-majorized positive linear functional on $\mathscr{A}$. Since $\phi$ is singular, we have $f^{\circ}=0$. Hence, $\pi(K)$ $=0$, and so $P_{\mathfrak{Y} \phi}^{\prime}=0$.

Let $(\mathscr{M}, \mathscr{D})$ be a closed $O_{p}^{*}$-algebra with the identity operator. Then, a positive invariant sesquilinear form $\phi$ on a $T^{+}$-algebra $\mathfrak{U}=\mathscr{M} \times \mathscr{D} \times \mathscr{D}^{*}$ defined by $\phi(A, B)=(\lambda(A) \mid \lambda(B))$ for $A, B \in \mathfrak{U}$ is nondegenerate and singular, and the restriction $\phi_{0}$ of $\phi$ to $\mathfrak{U}_{0}$ is nilpotent, where $\mathfrak{A}_{0}=\{0\} \times \mathscr{D} \times \mathscr{D}^{*}$. Tomita has given in [28] an example of a singular positive invariant sesquilinear form on the Schwartz space $\mathscr{S}\left(\boldsymbol{R}^{n} \times \boldsymbol{R}^{n}\right)$ which is regarded as the topological *-algebra of continuous operators in $L^{2}\left(\boldsymbol{R}^{n}\right)$.

We proceed to a decomposition theorem of positive invariant sesquilinear forms. Let $\phi$ be a positive invariant sesquilinear form on $\mathscr{A} \times \mathscr{A}$. We denote by $P(\phi)$ the set of all positive invariant sesquilinear forms $\psi$ on $\mathscr{A} \times \mathscr{A}$ with $\psi \leqq \phi$. Then $(P(\phi), \leqq)$ is an ordered set. We denote by $P_{r}(\phi)$ (resp. $\left.P_{s}(\phi), P_{n s}(\phi), P_{n}(\phi)\right)$ the set of all regular (resp. singular, nondegenerate and singular, nilpotent) positive invariant sesquilinear forms $\psi$ on $\mathscr{A} \times \mathscr{A}$ with $\psi \leqq \phi$.

Theorem 6.3. If $\phi$ is a positive invariant sesquilinear form on $\mathscr{A} \times \mathscr{A}$ satisfying $\pi_{\phi}^{*}=\pi_{\phi}^{* *}$, then there exists a decomposition $\phi=\phi_{r}+\phi_{s}$ of $\phi$ such that $\phi_{r} \in P_{r}(\phi), \phi_{s} \in P_{s}(\phi)$ and $\phi_{r}$ is maximal in the ordered set $\left(P_{r}(\phi), \leqq\right)$. In particular, if $\phi$ is a Riesz form, then the above $\phi_{s}$ is decomposed into the sum $\phi_{s}=\phi_{n s}+\phi_{n}$, where $\phi_{n s} \in P_{n s}(\phi)$ and $\phi_{n} \in P_{n}(\phi)$. Furthermore, the above $\phi_{r}, \phi_{s}$, $\phi_{n s}$ and $\phi_{n}$ are Riesz forms.

To prove the above theorem we prepare the following lemma.

LEMMA 6.4. Let $\phi, \psi$ be positive invariant sesquilinear forms on $\mathscr{A} \times \mathscr{A}$ such that $\psi \leqq \phi . \quad$ Put

$$
K_{\phi, \psi} \lambda_{\phi}(x)=\lambda_{\psi}(x)
$$

for $x \in \mathscr{A}$. Then the following statements hold.

(1) $K_{\phi, \psi}$ is extended to a continuous linear transformation of $\mathscr{S}_{\dot{\phi}}$ into $\mathfrak{S}_{\psi}$, which is also denoted by $K_{\phi, \psi}$.

(2) $T_{\phi, \psi}=K_{\phi, \psi}^{*} K_{\phi, \psi} \in \pi_{\phi}(\mathscr{A})^{\prime}$. 
(3) $K_{\phi, \psi}^{*} \mathscr{D}\left(\pi_{\psi}^{*}\right) \subset \mathscr{D}\left(\pi_{\phi}^{*}\right)$ and $\pi_{\phi}^{*}(x) K_{\phi, \psi}^{*} \eta=K_{\phi, \psi}^{*} \pi_{*}^{*}(x) \eta$ for each $x \in \mathscr{A}$ and $\eta \in \mathscr{D}\left(\pi_{*}^{*}\right)$.

(4) $K_{\phi, \psi} \mathscr{D}\left(\pi_{\phi}^{* *}\right) \subset \mathscr{D}\left(\pi_{\psi}^{*}\right)$ and $\pi_{\psi}^{*}(x) K_{\phi, \psi} \xi=K_{\phi, \psi} \pi_{\phi}^{* *}(x) \xi$ for each $x \in \mathscr{A}$ and $\xi=\mathscr{D}\left(\pi_{\phi}^{* *}\right)$.

(5) If $\psi$ is regular, then $P_{\Re^{\prime} \phi} T_{\phi, \psi}=T_{\phi, \psi}$.

Proof. (1), (2) This is trivial.

(3) This follows from the equality:

$$
\begin{aligned}
\left(\pi_{\dot{\phi}}(x) \lambda_{\dot{\phi}}(y) \mid K_{\phi, \psi}^{*} \eta\right) & =\left(\pi_{\psi}(x) \lambda_{\psi}(y) \mid \eta\right) \\
& =\left(\lambda_{\dot{\phi}}(y) \mid K_{\phi, \psi}^{*} \pi_{\psi}(x)^{*} \eta\right)
\end{aligned}
$$

for each $x, y \in \mathscr{A}$ and $\eta \in \mathscr{D}\left(\pi_{*}^{*}\right)$.

(4) This follows immediately from the statement (3).

(5) Since $\psi$ is regular, there exists a net $\left\{K_{\alpha}\right\}$ in $\mathfrak{H}_{\psi}^{\prime}$ such that $0 \leqq \pi\left(K_{\alpha}\right)$ $\leqq I$ and $\left\{\pi\left(K_{\alpha}\right)\right\}$ converges weakly to $I$. For each $\alpha$ and $x, y \in \mathscr{A}$ it follows from the statement (3) that

$$
\begin{aligned}
K_{\phi, \psi}^{*} \pi\left(K_{\alpha}\right) K_{\phi, \psi} \pi_{\dot{\phi}}(x) \lambda_{\dot{\phi}}(y) & =K_{\phi, \psi}^{*} \pi\left(K_{\alpha}\right) \pi_{\psi}(x) \lambda_{\psi}(y) \\
& =K_{\phi, \psi}^{*} \pi_{, \psi}^{*}(x) \pi\left(K_{\alpha}\right) \lambda_{\psi}(y) \\
& =\pi_{\phi}^{*}(x) K_{\phi, \psi}^{*} \pi\left(K_{\alpha}\right) K_{\phi, \psi} \lambda_{\phi}(y)
\end{aligned}
$$

and

$$
\begin{aligned}
K_{\phi, \psi}^{*} \pi\left(K_{\alpha}\right) K_{\phi, \psi} \lambda_{\phi}(x) & =K_{\phi, \psi}^{*} \pi\left(K_{\alpha}\right) \lambda_{\psi}(x) \\
& =K_{\phi, \psi}^{*} \pi_{\psi}^{*}(x) \lambda\left(K_{\alpha}\right) \\
& =\pi_{\phi}^{*}(x) K_{\phi, \psi}^{*} \lambda\left(K_{\alpha}\right),
\end{aligned}
$$

which means that $\left(K_{\phi, \psi}^{*} \pi\left(K_{a}\right) K_{\phi, \psi}, K_{\phi, \psi}^{*} \lambda\left(K_{\alpha}\right),\left(K_{\phi, \psi}^{*} \lambda\left(K_{\alpha}\right)\right)^{*}\right) \in \mathfrak{Y _ { \phi } ^ { \prime }}$. Hence, we have

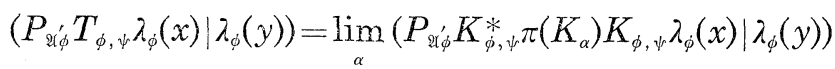

$$
\begin{aligned}
& =\lim _{\alpha}\left(K_{\dot{\phi}, \psi}^{*} \pi\left(K_{\alpha}\right) K_{\dot{\phi}, \psi} \lambda_{\dot{\phi}}(x) \mid \lambda_{\dot{\phi}}(y)\right) \\
& =\left(T_{\dot{\phi}, \psi} \lambda_{\phi}(x) \mid \lambda_{\dot{\phi}}(y)\right)
\end{aligned}
$$

for each $x, y \in \mathscr{A}$. Hence, $P_{9 \dot{q} \phi} T_{\phi, \psi}=T_{\phi, \psi}$.

Proof of Theorem 6.3. We put

$$
\begin{aligned}
& \phi_{r}(x, y)=\left(P_{x^{\prime} \dot{\phi}} \lambda_{\dot{\phi}}(x) \mid \lambda_{\dot{\phi}}(y)\right), \\
& \phi_{s}(x, y)=\left(\left(I-P_{x^{\prime} \dot{q}}\right) \lambda_{\phi}(x) \mid \lambda_{\phi}(y)\right)
\end{aligned}
$$

for $x, y \in \mathscr{A}$. Since $P_{\mathbb{q}^{\prime} \dot{\phi}} \in \pi_{\phi}(\mathscr{A})^{\prime}$, it is clear that $\phi_{r}, \phi_{s} \in P(\phi)$ and $\phi=\phi_{r}+\phi_{s}$. 


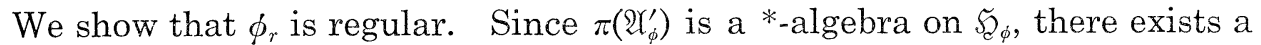
net $\left\{K_{\alpha}\right\}$ in $\mathfrak{Y}_{\dot{\phi}}^{\prime}$ such that $\left\|\pi\left(K_{\alpha}\right)\right\| \leqq 1$ for each $\alpha$ and $\left\{\pi\left(K_{\alpha}\right)\right\}$ converges strongly to $P_{\mathfrak{Q} \dot{q}^{\prime}}$. For each $x, y \in \mathscr{A}$ and $K \in \mathfrak{U}_{\phi}^{\prime}$ we have

$$
\begin{aligned}
\left(\pi(K) P_{\text {थ⿱㇒}}^{\prime} \lambda_{\dot{\phi}}(x) \mid \lambda_{\phi}(y)\right) & =\left(\lambda_{\phi}(x) \mid P_{\Re \mid \phi^{\prime} \phi} \pi\left(K^{+}\right) \lambda_{\phi}(y)\right) \\
& =\left(\lambda_{\phi}(x) \mid \pi\left(K^{+}\right) \lambda_{\phi}(y)\right) \\
& =\left(\pi(K) \lambda_{\phi}(x) \mid \lambda_{\phi}(y)\right),
\end{aligned}
$$

and hence $\pi(K) P_{\text {\& } \phi^{\prime}}=\pi(K)$. We now put

$$
f_{\alpha}(x)=\left(\pi\left(K_{\alpha}\right) \lambda_{\phi}(x) \mid \lambda\left(K_{\alpha}\right)\right)
$$

for $x \in \mathscr{A}$. Then it follows from $\pi_{\phi}^{*}=\pi_{\phi}^{* *}$ that

$$
\begin{aligned}
& f_{\alpha}\left(x^{*} x\right)=\left\|\pi\left(K_{\alpha}\right) \lambda_{\phi}(x)\right\|^{2}=\left\|\pi\left(K_{\alpha}\right) P_{\mathfrak{q}^{\prime} \phi} \lambda_{\phi}(x)\right\|^{2} \\
& \leqq\left\|P_{2(\phi} \lambda_{\phi}(x)\right\|^{2} \\
&=\phi_{r}(x, x), \\
&\left|f_{\alpha}(x)\right|^{2} \leqq\left\|\lambda\left(K_{\alpha}\right)\right\|^{2} \phi_{r}(x, x)
\end{aligned}
$$

for all $x \in \mathscr{A}$, which means that $f_{\alpha}$ is a $\phi_{r}$-majorized positive linear functional on $\mathscr{A}$. Furthermore, since

$$
\begin{aligned}
& \lim _{\alpha} f_{\alpha}^{\circ}(x, y)=\lim _{\alpha}\left(\pi\left(K_{\alpha}\right) \lambda_{\phi}(x) \mid \pi\left(K_{\alpha}\right) \lambda_{\phi}(y)\right) \\
& =\left(P_{\text {शí }}^{\prime} \lambda_{\phi}(x) \mid \lambda_{\dot{\phi}}(y)\right) \\
& =\phi_{r}(x, y)
\end{aligned}
$$

for all $x, y \in \mathscr{A}$, it follows that $\phi_{r}$ is regular.

We next show that $\phi_{s}$ is singular. Suppose that $\phi_{s}$ is not singular; that is, there exists a $\phi_{s}$-majorized positive linear functional $f$ on $\mathscr{A}$ such that $f^{\circ} \neq 0$. Since $\phi_{s} \leqq \phi, f$ is also $\phi$-majorized. Hence, there exists an element $K$ of $\mathfrak{Y}_{\dot{\phi}}^{\prime}$ such that $\pi(K) \neq 0$ and $f(x)=\left(\lambda_{\phi}(x) \mid \lambda(K)\right)$ for all $x \in \mathscr{A}$. Since $f^{\circ}$ is $\phi_{s}$-dominated, we have

$$
\left\|\pi(K) \lambda_{\dot{\phi}}(x)\right\|^{2}=f\left(x^{*} x\right) \leqq \phi_{s}(x, x)=\left\|\left(I-P_{2(\dot{\phi})}\right) \lambda_{\phi}(x)\right\|^{2}
$$

for all $x \in \mathscr{A}$, and hence

$$
\|\pi(K) \xi\| \leqq\left\|\left(I-P_{\text {qli }}^{\prime}\right) \xi\right\|
$$

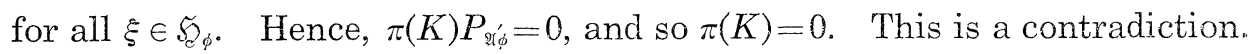
Hence, $\phi_{s}$ is singular.

Take arbitrary $\psi \in P_{r}(\phi)$. It follows from Lemma 6.4, (5) that

$$
\begin{aligned}
& \psi(x, x)=\left(T_{\phi, \psi} \lambda_{\phi}(x) \mid \lambda_{\dot{\phi}}(x)\right)=\left\|K_{\phi, \psi} P_{\mathscr{2} \dot{\phi}} \lambda_{\phi}(x)\right\|^{2} \\
& \leqq\left\|P_{\text {शं }}^{\prime} \lambda_{\phi}(x)\right\|^{2} \\
& =\phi_{r}(x, x)
\end{aligned}
$$


for all $x \in \mathscr{A}$, which implies that $\phi_{r}$ is maximal in the ordered set $\left(P_{r}(\phi), \leqq\right)$.

Suppose that $\phi$ is a Riesz form on $\mathscr{A}$. We denote by $P_{\mathfrak{r}_{\phi}}$ the projection

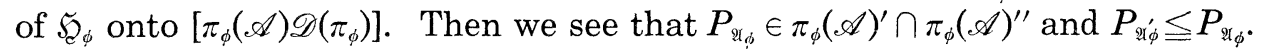
We now put

$$
\begin{aligned}
& \phi_{n s}(x, y)=\left(P_{\mathscr{2}_{\phi}}\left(I-P_{\mathfrak{q}_{\phi}^{\prime}}\right) \lambda_{\phi}(x) \mid \lambda_{\phi}(y)\right), \\
& \phi_{n}(x, y)=\left(\left(I-P_{\mathscr{q}_{\phi}}\right) \lambda_{\dot{\phi}}(x) \mid \lambda_{\phi}(y)\right)
\end{aligned}
$$

for $x, y \in \mathscr{A}$. Then it is clear that $\phi_{n s}, \phi_{n} \in P\left(\phi_{s}\right)$ and $\phi_{s}=\phi_{n s}+\phi_{n}$. As for the case of $\phi_{r}$, $\phi_{s}$, we can prove that $\phi_{n s}$ is nondegenerate and singular, and $\phi_{n}$ is nilpotent. We see immediately that $\phi_{r}, \phi_{s}, \phi_{n s}$ and $\phi_{n}$ are Riesz forms. This completes the proof.

COROLLARY 6.5. Let $\phi_{1}$ and $\phi_{2}$ be regular positive invariant sesquilinear forms on $\mathscr{A} \times \mathscr{A}$. Suppose that $\phi=\phi_{1}+\phi_{2}$ satisfies $\pi_{\phi}^{*}=\pi_{\phi}^{* *}$. Then $\phi$ is regular. Hence, if $\phi_{1}$ and $\phi_{2}$ are regular admissible positive invariant sesquilinear forms, then $\phi_{1}+\phi_{2}$ is regular.

Proof. By Lemma 6.4 there exist positive operators $T_{1}, T_{2}$ in $\pi_{\phi}(\mathscr{A})^{\prime}$ such that $T_{i} P_{\mathscr{Q}^{\prime} \phi}=T_{i}$ and $\phi_{i}(x, y)=\left(T_{i} \lambda_{\phi}(x) \mid \lambda_{\phi}(y)\right)$ for all $x, y \in \mathscr{A}(i=1,2)$. Since $\phi=\phi_{1}+\phi_{2}$, we have $T_{1}+T_{2}=I$, which implies that

$$
\begin{aligned}
& \phi(x, y)=\phi_{1}(x, y)+\phi_{2}(x, y)
\end{aligned}
$$

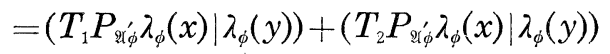

$$
\begin{aligned}
& =\left(P_{\text {q }{ }^{\prime} \lambda_{\dot{\phi}}}(x) \mid \lambda_{\dot{\phi}}(y)\right) \\
& =\phi_{r}(x, y)
\end{aligned}
$$

for all $x, y \in \mathscr{A}$. Hence, $\phi=\phi_{r}$, and so $\phi$ is regular.

We don't know whether the decomposition $\phi=\phi_{r}+\phi_{s}$ of Theorem 6.3 is unique, or not. However, we next show that the decomposition is unique in the case of commutative $*_{\text {-algebras. }}$

TheOREM 6.6. Every Riesz form $\phi$ on a commutative *-algebra $\mathscr{A}$ is uniquely decomposed into the sum $\phi=\phi_{r}+\phi_{s}$ of the regular Riesz form $\phi_{r}$ and the singular Riesz form $\phi_{s}$.

Proof. We show the uniqueness of such a decomposition. Suppose that $\phi=\phi_{1}+\phi_{2}$, where $\phi_{1} \in P_{r}(\phi)$ and $\phi_{2} \in P_{s}(\phi)$. Since $\phi_{1} \leqq \phi_{r}$ by Theorem 6.3, it follows that $\phi_{r}-\phi_{1} \in P\left(\phi_{r}\right), \phi_{2}-\phi_{s} \in P\left(\phi_{2}\right)$ and $\phi_{r}-\phi_{1}=\phi_{2}-\phi_{s}$. By Lemma 5.5, $\phi_{r}-\phi_{1}$ is regular and $\phi_{2}-\phi_{s}$ is singular. Hence, $\phi_{r}=\phi_{1}$ and $\phi_{s}=\phi_{2}$. This completes the proof.

REMARK. A positive linear functional $f$ on a ${ }^{*}$-algebra satisfying $\pi_{f}^{*}=$ 
$\pi_{f}^{* *}$ is decomposed into the sum $f=f_{r}+f_{s}$ of a regular positive linear functional $f_{r}$ and a singular positive linear functional. If $f$ is a Riesz functional on a commutative *-algebra, then the above decomposition is unique. In fact, since $\pi\left(\mathfrak{H}_{f}^{\prime}\right)$ is a ${ }^{*}$-algebra on $\mathfrak{S}_{f}$, there exists a net $\left\{K_{\alpha}\right\}$ in $\mathfrak{Y}_{f}^{\prime}$ such that $\left\|\pi\left(K_{\alpha}\right)\right\| \leqq 1$ for each $\alpha$ and $\left\{\pi\left(K_{\alpha}\right)\right\}$ converges strongly to $P_{\text {r }^{\prime}}$. We now put

$$
f_{r}(x)=\lim _{\alpha}\left(\pi\left(K_{\alpha}\right) \lambda_{f}(x) \mid \lambda\left(K_{\alpha}\right)\right), \quad f_{s}(x)=f(x)-f_{r}(x)
$$

for $x \in \mathscr{A}$. Then we have

$$
f_{r}\left(y^{*} x\right)=\left(P_{\mathfrak{Y}^{\prime}} \lambda_{f}(x) \mid \lambda_{f}(y)\right), \quad f_{s}\left(y^{*} x\right)=\left(\left(I-P_{\mathfrak{Y}^{\prime} f}\right) \lambda_{f}(x) \mid \lambda_{f}(y)\right)
$$

for all $x, y \in \mathscr{A}$. Hence, it follows from the proof of Theorem 6.3 that $f_{r}$ is a regular positive linear functional and $f_{s}$ is a singular positive linear functional.

\section{§. Applications.}

In this section we investigate weights on $C^{*}$-algebras by applying our study.

Let $\mathscr{A}$ be a $C^{*}$-algebra and $\mathscr{A}_{+}$be the positive part of $\mathscr{A}$. A function $\phi$ of $\mathscr{A}_{+}$into $[0, \infty]$ is said to be a weight on $\mathscr{A}$ if

(1) $\phi(x+y)=\phi(x)+\phi(y) \quad$ for $x, y \in \mathscr{A}_{+}$,

(2) $\phi(\lambda x)=\lambda \phi(x) \quad$ for $\lambda \geqq 0$ and $x \in \mathscr{A}_{+}$,

where $0 \cdot \infty=0$. Let $\phi$ be a weight on $\mathscr{A}$. We denote by $\mathfrak{R}_{\phi}$ the $\operatorname{set}\{x \in \mathscr{A}$; $\left.\phi\left(x^{*} x\right)<\infty\right\}$ and denote by $\mathfrak{M}_{\phi}$ the ${ }^{*}$-algebra $\mathfrak{N}_{\phi}^{*} \mathfrak{N}_{\phi}$. It is well known in the theory of weights that $\mathfrak{M}_{\phi}$ is a left ideal in $\mathscr{A}$ and $\mathfrak{M}_{\dot{\phi}}$ is a *-subalgebra spanned by $\mathscr{D}(\phi)=\left\{x \in \mathscr{A}_{+} ; \phi(x)<\infty\right\}$. The weight $\phi$ has a unique extension, which we still denote by $\phi$, to a linear functional on $\mathfrak{M}_{\phi}$. We now put

$$
\phi^{\circ}(x, y)=\phi\left(y^{*} x\right)
$$

for $x, y \in \mathfrak{N}_{\phi}$. Then $\phi^{\circ}$ is an admissible positive invariant sesquilinear form on $\mathfrak{N}_{\dot{\phi}} \times \mathfrak{N}_{\dot{\phi}}$. We show later on that for the positive invariant sesquilinear form $\phi^{\circ}$ generated by a weight $\phi$ on a $C^{*}$-algebra the regularity and the $\Sigma$ regularity are equivalent. We denote by $\left(\pi_{\phi}, \lambda_{\phi}, \mathfrak{S}_{\dot{\phi}}\right)$ the GNS-construction of $\phi^{\circ}$. Since $\mathfrak{R}_{\phi}$ is a left ideal in $\mathscr{A}$, the representation $\pi_{\phi}$ of $\mathfrak{R}_{\phi}$ implies a representation of $\mathscr{A}$ on $\tilde{S}_{\dot{\phi}}$ (again denoted by $\pi_{\dot{\phi}}$ ) by

$$
\pi_{\phi}(a) \lambda_{\phi}(x)=\lambda_{\dot{\phi}}(a x)
$$

for $a \in \mathscr{A}$ and $x \in \mathfrak{R}_{\phi}$.

Definition 7.1. Let $\phi_{1}, \phi_{2}$ be weights on a $C^{*}$-algebra $\mathscr{A}$. If $\phi_{1}(x) \leqq \phi_{2}(x)$ 
for each $x \in \mathscr{A}_{+}$, then we say that $\phi_{1}$ is dominated by $\phi_{2}$, which is denoted by $\phi_{1} \leqq \phi_{2}$. If $\mathscr{D}\left(\phi_{2}\right) \supset \mathscr{D}\left(\phi_{1}\right)$ and $\phi_{1}(x)=\phi_{2}(x)$ for all $x \in \mathscr{D}\left(\phi_{1}\right)$, then we say that $\phi_{2}$ is an extension of $\phi_{1}$, which is denoted by $\phi_{1} \subset \phi_{2}$.

Let $\left\{f_{\alpha}\right\}$ be a set of positive linear functionals of a $C^{*}$-algebra $\mathscr{A}$. We note that $\sum_{\alpha} f_{\alpha}$ and $\sup _{\alpha} f_{\alpha}$ are weights on $\mathscr{A}$.

DeFinition 7.2. Let $\phi$ be a weight on a $C^{*}$-algebra $\mathscr{A}$. If there exists a positive linear functional $f$ on $\mathscr{A}$ such that $f \supset \phi$, then $\phi$ is called strongly regular. If there exists a set $\left\{f_{\alpha}\right\}$ of positive linear functionals on $\mathscr{A}$ such that $\sum_{\alpha} f_{\alpha} \supset \phi$ (resp. $\sup _{\alpha} f_{\alpha} \supset \phi$ ), then $\phi$ is $\Sigma$-regular (resp. regular). If there does not exist any positive linear functional $f$ on $\mathscr{A}$ such that the restriction $f \mid \mathscr{D}(\phi)$ of $f$ to $\mathscr{D}(\phi)$ is non-zero and $f \leqq \phi$, then $\phi$ is called singular.

We note that a weight $\phi$ on a $C^{*}$-algebra is strongly regular if and only if the positive invariant sesquilinear form $\phi^{\circ}$ on $\mathfrak{R}_{\phi} \times \mathfrak{N}_{\phi}$ is strongly regular. For the regularity and the $\Sigma$-regularity of a weight on a $C^{*}$-algebra we have the following result.

TheORem 7.3. Let $\phi$ be a weight on a $C^{*}$-algebra $\mathscr{A}$. Then the following statements are equivalent.

(1) $\phi$ is regular.

(2) $\phi^{\circ}$ is a regular positive invariant sesquilinear form on $\mathfrak{A}_{\dot{\phi}} \times \mathfrak{N}_{\dot{\phi}}$.

(3) $\phi$ is $\Sigma$-regular.

(4) $\phi^{\circ}$ is a $\Sigma$-regular positive invariant sesquilinear form on $\mathfrak{N}_{\phi} \times \mathfrak{N}_{\phi}$.

(5) $\phi$ is uniformly lower semicontinuous on $\mathscr{D}(\phi)$.

Proof. $\quad(1) \Rightarrow(2) \quad$ It is sufficient to prove $P_{\mathfrak{Y}_{\phi}^{\prime}}=I$, where $\mathfrak{U}_{\phi}^{\prime}$ denotes the commutant of the $T^{+}$-algebra $\mathfrak{A}_{\phi}$ generated by $\phi^{\circ}$. Suppose that $\left(I-P_{\mathfrak{x}^{\prime} \dot{\phi}}\right) \lambda_{\phi}(a)$ $\neq 0$ for some $a \in \mathfrak{N}_{\phi}$. Since $\phi$ is regular, for each $\varepsilon>0$ there exists a positive linear functional $f$ on $\mathscr{A}$ such that $f \leqq \phi$ and $f\left(a^{*} a\right)+\varepsilon>\phi\left(a^{*} a\right)$. Then we note that $f$ is $\phi^{\circ}$-majorized. Hence, there exists an element $K$ of $\mathfrak{U}_{\dot{\phi}}^{\prime}$ such that $0 \leqq \pi(K) \leqq I$ and $f(x)=\left(\lambda_{\phi}(x) \mid \lambda(K)\right)$ for each $x \in \mathfrak{N}_{\phi}$. Furthermore, since $\pi(K) P_{\mathscr{x} \dot{\prime}}=\pi(K)$, it follows that

$$
\begin{aligned}
\left(P_{\Re \mid \dot{\phi}^{\prime}} \lambda_{\phi}(a) \mid \lambda_{\phi^{\prime}}(a)\right)+\varepsilon & \geqq\left(\pi(K) P_{\mathfrak{Y}^{\prime} \lambda_{\dot{\phi}}}(a) \mid \lambda_{\dot{\phi}}(a)\right)+\varepsilon \\
& =\left(\pi(K) \lambda_{\phi}(a) \mid \lambda_{\phi}(a)\right)+\varepsilon \\
& =f\left(a^{*} a\right)+\varepsilon \\
& >\phi\left(a^{*} a\right)=\left\|\lambda_{\phi}(a)\right\|^{2},
\end{aligned}
$$

so that $\left\|\left(I-P_{\mathfrak{R}^{\prime} \dot{\phi}}\right) \lambda_{\dot{\phi}}(a)\right\|<\varepsilon$. Hence, $\left(I-P_{\mathfrak{q}^{\prime} \dot{\phi}}\right) \lambda_{\dot{\phi}}(a)=0$, which is a contradiction. Hence, $P_{\mathfrak{x} \dot{\phi}}=I$.

$(2) \Rightarrow(3)$ By Proposition 5.2 there exists a net $\left\{K_{\alpha}\right\}$ in $\mathfrak{\mathfrak { V } _ { \phi } ^ { \prime }}$ such that 
$\left\|\pi\left(K_{\alpha}\right)\right\| \leqq 1$ for each $\alpha$ and $\left\{\pi\left(K_{\alpha}\right)\right\}$ converges strongly to $I$. Then we have

$$
\phi(x)=\sup _{\alpha} f_{\alpha}(x)
$$

for each $x \in \mathscr{D}(\phi)$, where $f_{\alpha}(a)=\left(\pi_{\dot{\phi}}(a) \lambda\left(K_{\alpha}\right) \mid \lambda\left(K_{\alpha}\right)\right)$ for $a \in \mathscr{A}$. We denote by $\tilde{\mathscr{A}}$ the universal enveloping von Neumann algebra of $\mathscr{A}$. If $f$ is a positive linear functional on $\mathscr{A}$, then there exists a unique normal positive linear functional $\tilde{f}$ on $\tilde{\mathscr{A}}$ which is an extension of $f$. We now put

$$
\tilde{\phi}(x)=\sup _{\alpha} \tilde{f}_{\alpha}(x)
$$

for $x \in \tilde{\mathscr{A}}$. Then $\tilde{\phi}$ is a normal weight on the von Neumann algebra $\tilde{\mathscr{A}}$ which is an extension of $\phi$. By ([21] Theorem 7.3) there exists a set $\left\{\omega_{\lambda}\right\}$ of normal positive linear functionals on $\tilde{\mathscr{A}}$ such that $\tilde{\phi}(x)=\sum_{\lambda} \omega_{\lambda}(x)$ for each $x \in \tilde{\mathscr{A}}_{+}$. Hence, we have

$$
\phi(x)=\tilde{\phi}(x)=\sum_{\lambda} \omega_{\lambda}(x)
$$

for each $x \in \mathscr{D}(\phi)$, which implies that $\phi$ is $\Sigma$-regular.

(3) $\Rightarrow(4)$ Suppose $\sum_{\alpha} f_{\alpha} \supset \phi$, where $\left\{f_{\alpha}\right\}$ is a set of positive linear functionals on $\mathscr{A}$. It is well known that every positive linear functional on a $C^{*}$-algebra is strongly regular. It hence follows that $f_{\alpha}$ is a $\phi^{\circ}$-majorized positive linear functional on $\mathfrak{N}_{\phi}$ and $\sum_{\alpha} f_{\alpha}^{\circ}(x, y)=\phi^{\circ}(x, y)$ for each $x, y \in \mathfrak{N}_{\phi}$, so that $\phi^{\circ}$ is $\Sigma$-regular.

$(4) \Rightarrow(5) \quad$ Since $\phi^{\circ}$ is $\Sigma$-regular, there exists a subset $\left\{\xi_{\alpha}\right\}$ of $\mathscr{S}_{\dot{\phi}}$ such that $\phi^{\circ}(x, y)=\sum_{\alpha}\left(\pi_{\phi}(x) \xi_{\alpha} \mid \pi_{\phi}(y) \xi_{\alpha}\right)$ for each $x, y \in \mathfrak{N}_{\phi}$. We now put

$$
f_{\alpha}(x)=\left(\pi_{\phi}(x) \xi_{\alpha} \mid \xi_{\alpha}\right)
$$

for $x \in \mathscr{A}$. Then $f_{\alpha}$ is a positive linear functional on $\mathscr{A}$ and $\sum_{\alpha} f_{\alpha} \supset \phi$. We note that the weight $\sum_{\alpha} f_{\alpha}$ on $\mathscr{A}$ is uniformly lower semicontinuous. Hence, $\phi$ is uniformly lower semicontinuous on $\mathscr{D}(\phi)$.

$(5) \Rightarrow(1) \quad$ This follows from ([4] Proposition 1.7).

The following corollary follows from the equivalence of (1) and (5) in Theorem 7.3.

CoRollary 7.4. If $\phi_{1}$ and $\phi_{2}$ are regular weights on a $C^{*}$-algebra $\mathscr{A}$, then $\phi_{1}+\phi_{2}$ is a regular weight on $\mathscr{A}$.

Proposition 7.5. Let $\phi$ be a weight on a $C^{*}$-algebra $\mathscr{A}$. Then $\phi$ is singular if and only if $\phi^{\circ}$ is a singular positive invariant sesquilinear form on $\mathfrak{N}_{\phi} \times \mathfrak{N}_{\phi}$.

Proof. Suppose that $\phi^{\circ}$ is not singular. Then there exists a $\phi^{\circ}$ - 
majorized positive linear functional $g$ on $\Re_{\phi}$ such that $g / \mathscr{D}(\phi) \neq 0$. By Lemma 4.2 there exists an element $K$ of $\mathfrak{Y}_{\phi}^{\prime}$ such that $\pi(K) \neq 0$ and $g(x)=\left(\lambda_{\phi}(x) \mid \lambda(K)\right)$ for all $x \in \mathfrak{R}_{\dot{\phi}}$, where $\mathfrak{H}_{\dot{\phi}}$ is a $T^{+}$-algebra generated by $\phi^{\circ}$. We now define a positive linear functional $f$ on $\mathscr{A}$ by

$$
f(x)=\frac{1}{\|\pi(K)\|^{2}}\left(\pi_{\phi}(x) \lambda(K) \mid \lambda(K)\right)
$$

for $x \in \mathscr{A}$. Then we have

$$
\begin{aligned}
f\left(x^{*} x\right) & =\frac{1}{\|\pi(K)\|^{2}}\left\|\pi_{\phi}(x) \lambda(K)\right\|^{2} \\
& =\frac{1}{\|\pi(K)\|^{2}}\left\|\pi(K) \lambda_{\dot{\phi}}(x)\right\|^{2} \\
& \leqq \phi\left(x^{*} x\right)
\end{aligned}
$$

for all $x \in \mathfrak{N}_{\phi}$ and $f / \mathscr{D}(\phi) \neq 0$ by $\pi(K) \neq 0$, which implies that $\phi$ is not singular.

Suppose that $\phi$ is not singular; that is, there exists a positive linear functional $f$ on $\mathscr{A}$ such that $f / \mathscr{D}(\phi) \neq 0$ and $f \leqq \phi$. Then we see that $f / \Re_{\phi}$ is a $\phi^{\circ}$-majorized positive linear functional on $\Re_{\phi}$ and $\left(f / \Re_{\phi}\right)^{\circ} \neq 0$, which implies that $\phi^{\circ}$ is not singular. This completes the proof.

We finally show a decomposition theorem for a weight $\phi$ on a $C^{*}$-algebra by making use of the decomposition theorem (Theorem 6.3) for the positive invariant sesquilinear form $\phi^{\circ}$.

Theorem 7.6. Let $\phi$ be a weight on a $C^{*}$-algebra $\mathscr{A}$. Then there exist a regular weight $\phi_{r}$ on $\mathscr{A}$ and a singular weight $\phi_{s}$ on $\mathscr{A}$ such that $\phi=\phi_{r}+\phi_{s}$ and $\phi_{r}$ is maximal in the ordered set $\left(W_{r}(\phi), \leqq\right)$, where $W_{r}(\phi)$ denotes the set of all regular weights $\psi$ on $\mathscr{A}$ with $\psi \leqq \phi$.

Proof. We denote by $\mathfrak{H}_{\phi}$ the $T^{+}$-algebra generated by the positive invariant sesquilinear form $\phi^{\circ}$ on $\mathfrak{N}_{\dot{\phi}} \times \mathfrak{N}_{\dot{\phi}}$. Since $\pi\left(\mathfrak{N}_{\phi}^{\prime}\right)$ is a $*$-algebra on $\mathfrak{H}_{\phi}$, there exists a set $\left\{K_{\alpha}\right\}$ in $\mathfrak{U}_{\phi}^{\prime}$ such that $\left\|\pi\left(K_{\alpha}\right)\right\| \leqq 1$ for each $\alpha$ and $\left\{\pi\left(K_{\alpha}\right)\right\}$ converges strongly to $P_{\text {r } q}^{\prime}$. For each $x \in \mathscr{A}_{+}$we put

$$
\phi_{r}(x)= \begin{cases}\lim _{\alpha}\left(\pi\left(K_{\alpha}\right) \lambda_{\phi}(x) \mid \lambda\left(K_{\alpha}\right)\right), & x \in \mathscr{D}(\phi) \\ \infty & , x \notin \mathscr{D}(\phi) .\end{cases}
$$

For each $x \in \mathscr{D}(\phi)$ we have

$$
\begin{aligned}
\phi_{r}(x) & =\lim _{\alpha}\left(\pi\left(K_{\alpha}\right) \lambda_{\dot{\phi}}(x) \mid \lambda\left(K_{\alpha}\right)\right) \\
& =\lim _{\alpha}\left\|\pi\left(K_{\alpha}\right) \lambda_{\phi}\left(x^{1 / 2}\right)\right\|^{2} \\
& =\left\|P_{\Re \mid \dot{\phi}} \lambda_{\phi}\left(x^{1 / 2}\right)\right\|^{2} .
\end{aligned}
$$


We show that $\phi_{r}(x+y)=\phi_{r}(x)+\phi_{r}(y)$ for each $x, y \in \mathscr{A}$. In fact, since $\phi(x+y)$ $=\phi(x)+\phi(y)$, it follows that $(x+y)^{1 / 2} \in \mathfrak{N}_{\dot{\phi}}$ if and only if $x^{1 / 2} \in \mathfrak{R}_{\dot{\phi}}$ and $y^{1 / 2} \in \mathfrak{R}_{\dot{\phi}}$. Hence we have that if $(x+y)^{1 / 2} \in \mathfrak{N}_{\phi}$ then

$$
\begin{aligned}
\phi_{r}(x+y) & =\lim _{\alpha}\left(\pi\left(K_{\alpha}\right) \lambda_{\phi}(x+y) \mid \lambda\left(K_{\alpha}\right)\right) \\
& =\lim _{\alpha}\left\{\left(\pi\left(K_{\alpha}\right) \lambda_{\phi}(x) \mid \lambda\left(K_{\alpha}\right)\right)+\left(\pi\left(K_{\alpha}\right) \lambda_{\phi}(y) \mid \lambda\left(K_{\alpha}\right)\right)\right\} \\
& =\phi_{r}(x)+\phi_{r}(y)
\end{aligned}
$$

and if $(x+y)^{1 / 2} \in \Re_{\phi}$, then $\phi_{r}(x+y)=\phi_{r}(x)+\phi_{r}(y)=\infty$. It is clear that $\phi_{r}(\lambda x)=$ $=\lambda \phi_{r}(x)$ for each $\lambda \geqq 0$ and $x \in \mathscr{A}_{+}$. Hence, $\phi_{r}$ is a weight on $\mathscr{A}$ with $\mathfrak{R}_{\phi_{r}}=\Re_{\dot{\phi}}$. For each $x, y \in \mathfrak{R}_{\dot{\phi}}$ we have

$$
\begin{aligned}
\phi_{r}^{\circ}(x, y) & =\lim _{\alpha}\left(\pi\left(K_{\alpha}\right) \lambda_{\phi}(x) \mid \pi\left(K_{\alpha}\right) \lambda_{\phi}(y)\right) \\
& =\left(P_{\mathscr{Y}^{\prime} \phi} \lambda_{\phi}(x) \mid \lambda_{\phi}(y)\right) .
\end{aligned}
$$

It hence follows from Theorem 6.3 that $\phi_{r}^{\circ}$ is a regular positive invariant sesquilinear form on $\mathfrak{N}_{\dot{\phi}} \times \mathfrak{N}_{\dot{\phi}}$, and so $\phi_{r}$ is regular by Theorem 7.3. For each $x \in \mathscr{A}^{+}$we put

$$
\phi_{s}(x)= \begin{cases}\phi(x)-\phi_{r}(x), & x \in \mathscr{D}(\phi) \\ \infty, & x \notin \mathscr{D}(\phi) .\end{cases}
$$

It follows since $\phi_{r} \leqq \phi$ that $\phi_{s}$ is a weight on $\mathscr{A}$ satisfying that $\Re_{\dot{\phi}_{s}}=\mathfrak{R}_{\dot{\phi}}$ and $\dot{\phi}_{s}^{\circ}(x, y)=\left(\left(I-P_{\mathfrak{Y}^{\prime} \dot{\phi}}\right) \lambda_{\dot{\phi}}(x) \mid \lambda_{\phi}(y)\right)$ for each $x, y \in \mathfrak{R}_{\phi^{\prime}}$. Hence, if follows from Theorem 6.3 and Proposition 7.5 that $\phi_{s}$ is singular and $\phi=\phi_{r}+\phi_{s}$.

Suppose that $\psi$ is a regular weight on $\mathscr{A}$ with $\psi \leqq \phi$. Since $\mathfrak{R}_{\phi} \subset \mathfrak{R}_{\psi}$, we see that the restriction $\psi^{\circ} / \Re_{\dot{\phi}} \times \mathfrak{N}_{\dot{\phi}}$ of the positive invariant sesquilinear form $\psi^{\circ}$ on $\mathfrak{N}_{\psi} \times \mathfrak{N}_{\psi}$ is a regular positive invariant sesquilinear form on $\mathfrak{N}_{\dot{\phi}} \times \mathfrak{N}_{\dot{\phi}}$. It hence follows from Theorem 6.3 that $\psi^{\circ} / \mathfrak{N}_{\dot{q}} \times \mathfrak{R}_{\dot{\phi}} \leqq \phi_{r}^{\circ}$, which implies $\psi \leqq \phi_{r}$. This completes the proof.

We remark that if $\phi$ is a weight on a $C^{*}$-algebra $\mathscr{A}$, then $\sup _{f \in \mathscr{F}} f \supset \phi_{r}$ by Theorem 7.6, where $\mathscr{F}$ denotes the set of all positive linear functionals $f$ on $\mathscr{A}$ with $f \leqq \phi$.

\section{References}

[1] G. R. Allan, A spectral theory for locally convex algebras, Proc. London Math. Soc., 15 (1965), 399-421.

[2] G. R. Allan, On a class of locally convex algebras, Proc. London Math. Soc., 17 (1967), 91-114.

[3] S. J. Bhatt, Representability of positive functionald on abstract star algebras 
without identity with applications to locally convex *-algebras, to appear in Yokohama Math. J., 29 (1981), 7-16.

[4] F. Combes, Poids sur une $C^{*}$-algèbre, J. Math. Pures Appl., 42 (1968), 57-100.

[ 5 ] J. Diximier, Les algèbres d'opérateurs dans l'espace Hilbertian, Gauthier-Villars, Paris, 2é edition, 1969.

[ 6 ] P. G. Dixon, Generalized $B^{*}$-algebras, Proc. London Math. Soc., 21 (1970), $693-$ 715 .

[7] I. M. Gelfand and N. Ya. Vilenkin, Generalized functions, Vol. 4., Academic Press, New York, 1964.

[8] R. Godement, Sur la theorie des représentations unitaires, Ann. of Math., 53 (1951), 68-124.

[ 9 ] S. P. Gudder and W. Scruggs, Unbounded representations of *algebras, Pacific J. Math., 70 (1977), 369-382.

[10] S. P. Gudder and R. L. Hudson, A noncommutative probability theory, Trans. Amer. Math. Soc., 245 (1978), 1-41.

[11] U. Haagerup, Normal weights on $W^{*}$-algebras, J. Functional Analysis, 19 (1975), 302-317.

[12] A. Inoue, Unbounded representations of symmetric *-algebras, J. Math. Soc. Japan, 29 (1977), 219-232.

[13] A. Inoue, Unbounded generalizations of left Hilbert algebras, J. Functional Analysis, 34 (1979), 339-362.

[14] A. Inoue, Unbounded generalizations of left Hilbert algebras II, J. Functional Analysis, 35 (1980), 230-250.

[15] A. Inoue and K. Takesue, Self-adjoint representations of polynomial algebras, to appear in Trans. Amer. Math. Soc. .

[16] G. Lassner, Topological algebras of operators, Rep. Mathematical Phys., 3 (1972), 279-293.

[17] L. H. Loomis, An introduction to abstract harmonic analysis, Van Nostrand, New York, 1953.

[18] M. A. Naimark, Normed algebras, 3rd. ed., Wolters-Noordhoff, Gröningen, 1972.

[19] A. E. Nussbaum, On the integral representation of positive linear functionals, Trans. Amer. Math. Soc., 128 (1967), 460-473.

[20] G. K. Pedersen, Measure theory for $C^{*}$-algebras, Math. Scand., 19 (1966), 131145.

[21] G. K. Pedersen and M. Takesaki, The Radon-Nikodym theorem for von Neumann algebras, Acta Math., 130 (1973), 53-87.

[22] R. T. Powers, Self-adjoint algebras of unbounded operators, Comm. Math. Phys., 21 (1971), 85-124.

[23] C.E. Rickart, General theory of Banach algebras, Van Nostrand, Princeton, 1960.

[24] S. Sakai, $C^{*}$-algebras and $W^{*}$-algebras, Springer-Verlag, Berlin and New York, 1971.

[25] K. Schmüdgen, On trace representation of linear functionals on unbounded operator algebras, Comm. Math. Phys., 63 (1978), 113-130.

[26] T. Sherman, Positive linear functionals of *algebras of unbounded operators, J. Math. Anal. Appl., 22 (1968), 285-318.

[27] M. Tomita, Standard forms of von Neumann algebras, The Fifth Functional Analysis Symposium of the Mathematical Society of Japan, Sendai, 1967.

[28] M. Tomita, Foundations of noncommutative Fourier analysis, Japan-U.S. Seminor on $C^{*}$-algebras and Applications to Physics, Kyoto, 1974.

[29] S. L. Woronowicz, The quantum problem of moments I, Rep. Mathematical Phys., 1 (1970), 135-145. 
[30] S. L. Woronowicz, The quantum problem of moments II, Rep. Mathematical Phys., 1 (1970), 175-183.

Atsushi Inoue

Department of Applied Mathematics

FACULTY OF SCIENCE

FUKUOKA UNIVERSITY

FUKUOKA 814-01

JAPAN 